\title{
Seismic and spectroscopic characterization of the solar-like pulsating CoRoT target HD 49385 ${ }^{\star}, \star \star$
}

\author{
S. Deheuvels ${ }^{1}$, H. Bruntt ${ }^{1}$, E. Michel ${ }^{1}$, C. Barban ${ }^{1}$, G. Verner $^{2}$, C. Régulo ${ }^{3,4}$, B. Mosser ${ }^{1}$, S. Mathur ${ }^{5}$, P. Gaulme ${ }^{6}$,

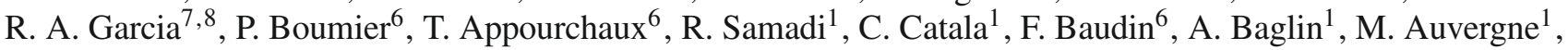 \\ I. W. Roxburgh ${ }^{1,2}$, and F. Pérez Hernández ${ }^{3,4}$ \\ 1 LESIA, UMR8109, Université Pierre et Marie Curie, Université Denis Diderot, Observatoire de Paris, 92195 Meudon Cedex, \\ France \\ e-mail: sebastien.deheuvels@obspm.fr \\ 2 Astronomy Unit, Queen Mary, University of London Mile End Road, London E1 4NS, UK \\ 3 Instituto de Astrofísica de Canarias, 38205, La Laguna, Tenerife, Spain \\ 4 Universidad de La Laguna, 38206 La Laguna, Tenerife, Spain \\ 5 Indian Institute of Astrophysics, Koramangala, Bangalore 560034, India \\ 6 Institut d'Astrophysique Spatiale, UMR8617, Université Paris XI, Bâtiment 121, 91405 Orsay Cedex, France \\ 7 Laboratoire AIM, CEA/DSM-CNRS-Université Paris Diderot; CEA, IRFU, SAp, centre de Saclay, 91191, Gif-sur-Yvette, France \\ ${ }^{8}$ GEPI, Observatoire de Paris, CNRS, Université Paris Diderot; 5 place Jules Janssen, 92190 Meudon, France
}

Received 16 October 2009 / Accepted 8 March 2010

\begin{abstract}
Context. The star HD 49385 is the first G-type solar-like pulsator observed in the seismology field of the space telescope CoRoT. The satellite collected 137 days of high-precision photometric data on this star, confirming that it presents solar-like oscillations. HD 49385 was also observed in spectroscopy with the NARVAL spectrograph in January 2009.

Aims. Our goal is to characterize HD 49385 using both spectroscopic and seismic data.

Methods. The fundamental stellar parameters of HD 49385 are derived with the semi-automatic software VWA, and the projected rotational velocity is estimated by fitting synthetic profiles to isolated lines in the observed spectrum. A maximum likelihood estimation is used to determine the parameters of the observed p modes. We perform a global fit, in which modes are fitted simultaneously over nine radial orders, with degrees ranging from $\ell=0$ to $\ell=3$ (36 individual modes).

Results. Precise estimates of the atmospheric parameters $\left(T_{\text {eff }},[\mathrm{M} / \mathrm{H}], \log g\right)$ and of the $v \sin i$ of $\mathrm{HD} 49385$ are obtained. The seismic analysis of the star leads to a clear identification of the modes for degrees $\ell=0,1,2$. Around the maximum of the signal $(v \simeq 1013 \mu \mathrm{Hz}$ ), some peaks are found significant and compatible with the expected characteristics of $\ell=3$ modes. Our fit yields robust estimates of the frequencies, linewidths and amplitudes of the modes. We find amplitudes of $\sim 5.6 \pm 0.8$ ppm for radial modes at the maximum of the signal. The lifetimes of the modes range from one day (at high frequency) to a bit more than two days (at low frequency). Significant peaks are found outside the identified ridges and are fitted. They are attributed to mixed modes.
\end{abstract}

Key words. methods: data analysis - methods: statistical - methods: observational - stars: oscillations - stars: individual: HD 49385

\section{Introduction}

In the Sun, oscillations are excited by the turbulent motions in the outer part of the external convective envelope and are further propagated into the interior of the star. The study of these oscillations has yielded constraints on the inner structure of the Sun, allowing us to estimate the sound speed and density profiles (Basu et al. 2003; Turck-Chièze et al. 2001), the position of the base of the convective zone (Christensen-Dalsgaard et al. 1991), and the rotation profile (Thompson et al. 2003; Mathur et al. 2008). However, the very low amplitude of these oscillations (a

* Based on data obtained from the CoRoT (Convection, Rotation and planetary Transits) space mission, developed by the French Space agency CNES in collaboration with the Science Programs of ESA, Austria, Belgium, Brazil, Germany and Spain.

$\star \star$ Based on data obtained using the Télescope Bernard Lyot at Observatoire du Pic du Midi, CNRS and Université Paul Sabatier, France. few ppm in photometry) makes it very challenging to detect and analyze them in other stars than the Sun.

Achieving a better understanding of the interiors of solarlike pulsations is one of the main objectives of the space mission CoRoT (Convection, Rotation and planetary Transits). CoRoT is a space telescope performing high-precision photometry over quasi-uninterrupted long observing runs (Baglin et al. 2006). Solar-like oscillations have already been studied in several other stars with CoRoT data. The analyses of these stars have encountered difficulties identifying the degrees of the modes, either because of a too low signal-to-noise ratio (HD 175726: Mosser et al. 2009b; HD 181906: García et al. 2009), or because of a too short lifetime of the modes, inducing large mode linewidths (HD 49933: Appourchaux et al. 2008; HD 181420: Barban et al. 2009).

The star HD 49385 is the first G-type solar-like pulsator observed in the seismology field of CoRoT. It is cooler than the solar-like pulsators previously analysed with CoRoT data, and probably more evolved (at the end of the main sequence or 
shortly after it). The choice of HD 49385 as a CoRoT target has motivated us to lead spectroscopic observations, performed with the NARVAL spectrograph at the Pic du Midi Observatory. The fundamental parameters of HD 49385 are derived from these observations, as described in Sect. 2. The photometric observations with CoRoT are presented in Sect. 3. Section 4 presents the study of the low-frequency part of the power spectrum, in search of a signature of the stellar rotation. The extraction of p-mode parameters is described in Sect. 5, and Sect. 6 is dedicated to conclusions.

\section{Stellar parameters of HD 49385}

The solar-like pulsator HD 49385 is a G0-type star with an apparent magnitude of $m_{\mathrm{V}}=7.39$ (uvby catalog, Hauck \& Mermilliod 1998). To determine the fundamental parameters of HD 49385 we analysed a high-quality spectrum from the NARVAL spectrograph mounted on the 2-m Bernard Lyot Telescope at the Pic du Midi Observatory. We stacked two spectra collected on 2009 January 10, totalizing an exposure time of $6600 \mathrm{~s}$. The spectrum was normalized by identifying continuum windows in a synthetic spectrum and fitting a low-order spline through these points. This was done order-by-order and we made sure the line depths agreed for the overlapping part of adjacent echelle orders. We measured a mean signal-to-noise $(\mathrm{S} / \mathrm{N})$ ratio of 600 in the continuum for several line-free regions in the range 5000-7000 $\AA$ for a data sampling of 2.7 data points per resolution element $(R=65000)$.

\subsection{Temperature, $\log g$ and metalicity}

We used the semi-automatic VWA software (Bruntt 2009) to fit synthetic profiles for more than 600 lines in the range from 4135 to $8545 \AA$. We used a differential approach, meaning all abundances are measured relative to exactly the same lines in the solar spectral atlas from Kurucz et al. (1984). This differential approach is described in more detail by Bruntt et al. (2008). As part of the analysis, we determined the atmospheric model parameters $T_{\mathrm{eff}}, \log g$, and the microturbulence. This was done by requiring that abundances determined from $\mathrm{Fe}$ I lines do not correlate with the equivalent width $(\mathrm{EW})$ or the excitation potential (EP). Furthermore we required that the same mean abundances are measured from the Fe I and Fe II lines ("ionization balance"). We estimated the uncertainty on the model parameters by adjusting them until the correlations of Fe I with EW or EP became significant or the ionization balance deviated (see Bruntt et al. 2008, for details). We stress that the uncertainties are strictly internal errors because the underlying assumption is that the model atmosphere represents the star. Any systematic error in the temperature profile or departures from local thermal equilibrium (LTE) will likely affect the results. We therefore quadratically added $50 \mathrm{~K}$ and 0.05 dex to the uncertainty on $T_{\text {eff }}$ and $\log g$. The spectroscopic parameters are listed in Table 1.

As an additional check of the surface gravity we fitted the wings of the pressure sensitive Ca lines at 6122.2 and $6162.2 \AA$. This was done by first adjusting the van der Waals constants to fit the solar spectrum for the canonical value $\log g=4.438$. The fitted values for the two lines for HD 49385 are $\log g=3.96 \pm 0.12$ and $4.05 \pm 0.06$, which agrees very well with the Fe I/Fe II ionization balance. The low value we obtain for the $\log g$ of HD 49385 indicates that it certainly is an evolved object.

The parameters agree well with the calibration of the Strömgren indices. From TEMPLOGG (Rogers 1995;
Table 1. Spectroscopic parameters of HD 49385.

\begin{tabular}{cc}
\hline \hline$T_{\text {eff }}$ & $6095 \pm 65 \mathrm{~K}$ \\
$\log g$ & $4.00 \pm 0.06$ \\
{$[\mathrm{M} / \mathrm{H}]$} & $+0.09 \pm 0.05 \mathrm{dex}$ \\
$v \sin i$ & $2.9_{-1.5}^{+1.0} \mathrm{~km} \mathrm{~s}^{-1}$ \\
\hline
\end{tabular}

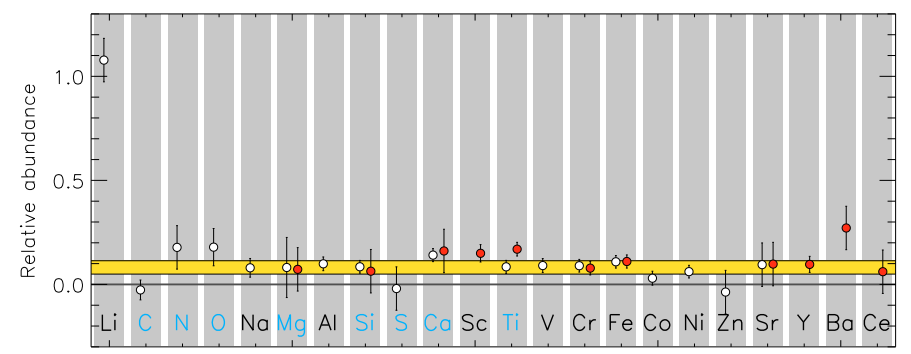

Fig. 1. Relative abundances of 22 elements measured in HD 49385. Open and solid circles are mean values for neutral and singly ionized lines, respectively. The yellow horizontal bar marks the mean metalicity with $1-\sigma$ uncertainty range.

Kupka \& Bruntt 2001) we get $T_{\text {eff }}=6345 \pm 150 \mathrm{~K}, \log g=$ $4.22 \pm 0.15$, and $[\mathrm{Fe} / \mathrm{H}]=+0.21 \pm 0.10$. TEMPLOGG determines a significant interstellar reddening $E(b-y)=0.020 \pm 0.007$. If we assume zero interstellar reddening, we get from the $V-K$, using the Ramírez \& Meléndez (2005) calibration, $T_{\text {eff }}=5958 \pm 64 \mathrm{~K}$ and $6035 \pm 92 \mathrm{~K}$ from $b-y$. The uncertainties include the calibration uncertainty and the photometric uncertainty on the indices. Finally, Kovtyukh et al. (2004) used line-depth ratios to determine effective temperatures of stars and found $T_{\text {eff }}=$ $6052 \pm 6.7 \mathrm{~K}$ (internal error). They also determined $\log g$ and $[\mathrm{Fe} / \mathrm{H}]$, but did not give uncertainties on these two parameters: $\log g=4.00$ and $[\mathrm{Fe} / \mathrm{H}]=+0.10$. In summary, our result from VWA agrees well with the photometric calibrations and the linedepth ratio method for $T_{\text {eff }}$.

We found a high abundance of lithium in HD 49385 of $+1.08 \pm 0.10$ (relative to the Sun). This value appears to be typical for a dwarf star with solar metalicity (e.g., Boesgaard et al. 2005). We used the line list from Ghezzi et al. (2009), but did not include the relatively weak molecular $\mathrm{CN}$ bands; hence the $\mathrm{Li}$ abundance may be slightly overestimated. The Li feature around 6707.8 is known to be strongly affected by NLTE effects, but when using 3D instead of 1D atmosphere models these two effects appear to cancel to first order (Asplund 2005). In other words, because we are using 1D LTE models, the imposed error should be relatively small. Furthermore, because we are calculating abundances relative to the same Li line feature as in the Sun, our estimate of [Li] should be robust as the atmospheric parameters of the two stars are quite similar.

The abundance pattern we determine is shown in Fig. 1 and listed in Table 2. The overall metalicity is determined as the mean of the metals with at least 10 lines used in the analysis ( $\mathrm{Si}, \mathrm{Ca}, \mathrm{Ti}, \mathrm{V}, \mathrm{Cr}, \mathrm{Fe}, \mathrm{Co}, \mathrm{Ni}$ ) giving $[\mathrm{M} / \mathrm{H}]=0.09 \pm 0.05$. This range is marked by the horizontal bar in Fig. 1.

\subsection{Projected rotational velocity}

Because we have a relatively high-resolution spectrum with very high $\mathrm{S} / \mathrm{N}$ we were able to estimate the projected rotational velocity $(v \sin i)$. We fitted synthetic profiles to ten isolated lines in the range 4600-6800 $\AA$. We calculated the synthetic profiles with the 


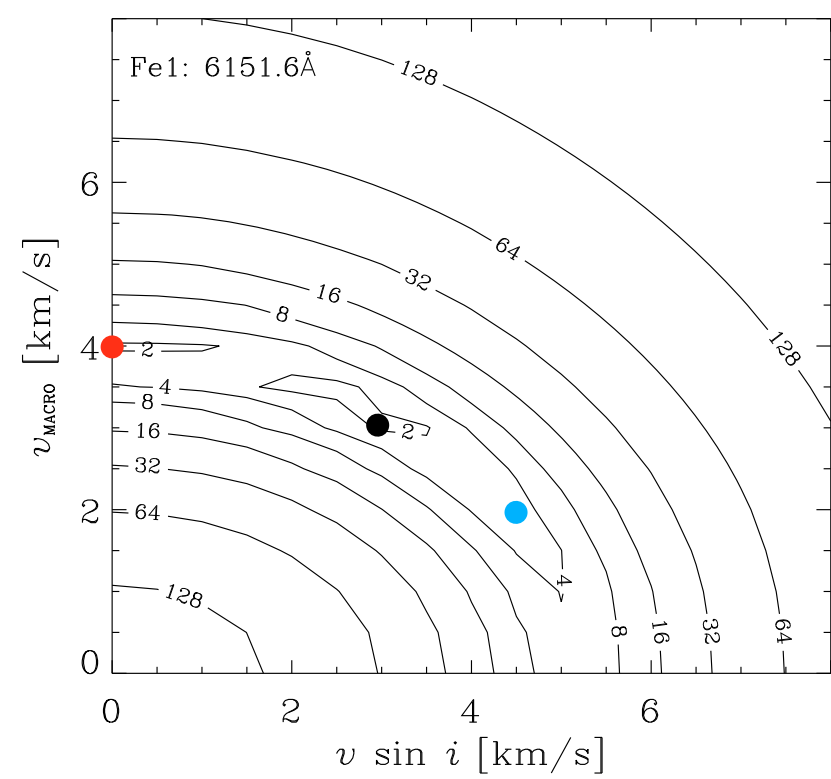

Fig. 2. Example of the $\chi^{2}$ contour surface used to determine $v \sin i$ and $v_{\text {macro }}$ for the $\mathrm{Fe}$ I line $\lambda 6151.6 \AA$. The three circles mark the minimum of the surface and the 1- $\sigma$ uncertainty range. The corresponding three synthetic spectra are compared to the observed line in Fig. 3.

SYNTH software Valenti \& Piskunov (1996), thus taking into account weak blends. We considered three broadening mechanisms which were convolved with the synthetic profiles: (1) the instrumental resolution; (2) macroturbulence $\left(v_{\text {macro }}\right)$; and (3) $v \sin i$. The instrumental power of resolution of NARVAL is $R=65000$, but to check this value we fitted six narrow telluric lines around $6290 \AA$. We found that a Gaussian function with a $F W H M$ of $6290 \AA / 65000=0.097 \AA$ fitted these lines very well and we used the same value of $R$ for the stellar lines. For $v \sin i$ and $v_{\text {macro }}$ we calculated a $17 \times 17$ grid of convolved profiles for $0-8 \mathrm{~km} \mathrm{~s}^{-1}$ in steps of $0.5 \mathrm{~km} \mathrm{~s}^{-1}$. To identify the best combination of $v \sin i$ and $v_{\text {macro }}$ we compared the synthetic profile and the observed spectrum by computing the $\chi^{2}$ value.

In Fig. 2 we show an example of the $\chi^{2}$ contour for the Fe I line at $6156.1 \AA$. The plots for all ten lines are very similar and show the same strong correlation between $v \sin i$ and $v_{\text {macro. We }}$ marked three points on the contour in Fig. 2 with filled circles, and the synthetic profiles corresponding to these grid points are shown in Fig. 3. The observed spectrum is shown with open circles. From our analysis we can put a firm upper limit on $v \sin i$ of $5 \mathrm{~km} \mathrm{~s}^{-1}$, corresponding to $v_{\text {macro }}=0 \mathrm{~km} \mathrm{~s}^{-1}$. The values that provide the best fit based on the analysis of the $\chi^{2}$ contours for all ten lines are $v \sin i=2.9_{-1.5}^{+1.0} \mathrm{~km} \mathrm{~s}^{-1}$ and $v_{\text {macro }}=3.4_{-0.7}^{+0.3} \mathrm{~km} \mathrm{~s}^{-1}$.

\subsection{Luminosity}

Using the Hipparcos parallax of the star, $\pi=13.91 \pm 0.76$ mas (van Leeuwen 2007), we could derive the absolute visual magnitude of the object $M_{\mathrm{V}}=3.11 \pm 0.12$. The bolometric correction was determined by interpolating in the grid provided by Bessell et al. (1998) for appropriate values of temperature and $\log g$ for HD 49385. We obtained $B C_{\mathrm{V}}=-0.029 \pm 0.006$, which yields a luminosity $\log \left(L / L_{\odot}\right)=0.67 \pm 0.05$. The values of $T_{\text {eff }}$ and $L$ provided an estimate of the radius $R / R_{\odot}=1.94 \pm 0.15$.

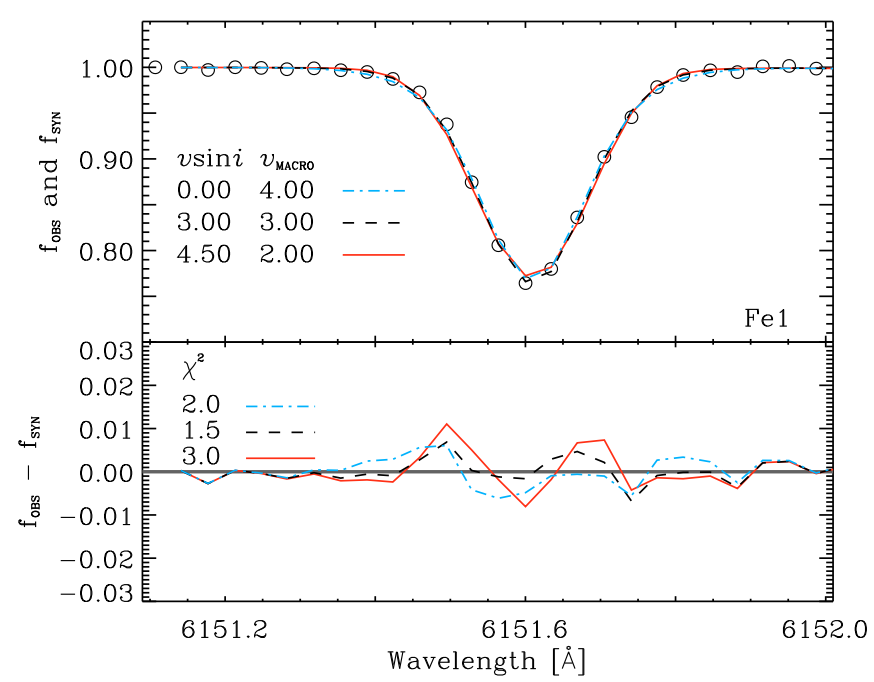

Fig. 3. The upper panel shows the fit of three synthetic profiles to the observed line (marked by open circles). Each profile was convolved with different combinations of $v \sin i$ and $v_{\text {macro }}$ as given in the top panel (cf. Fig. 2). The lower panel shows the difference between the observed and synthetic profiles, indicating a slightly better fit for the profile represented by a dashed line (the $\chi^{2}$ value for each fit is given in the lower panel).

Table 2. Abundances of 22 elements in HD 49385 relative to the Sun.

\begin{tabular}{llr|llr}
\hline \hline El. & $\Delta A$ & $n$ & El. & $\Delta A$ & $n$ \\
\hline C I & $-0.03 \pm 0.05$ & 9 & V I & $+0.09 \pm 0.03$ & 12 \\
N I & +0.18 & 1 & Cr I & $+0.09 \pm 0.03$ & 18 \\
O I & $+0.18 \pm 0.17$ & 4 & Cr II & $+0.08 \pm 0.03$ & 7 \\
Na I & $+0.08 \pm 0.04$ & 4 & Fe I & $+0.11 \pm 0.03$ & 336 \\
Mg I & $+0.08 \pm 0.14$ & 4 & Fe II & $+0.11 \pm 0.03$ & 27 \\
Mg II & $+0.07 \pm 0.03$ & 2 & Co I & $+0.03 \pm 0.03$ & 12 \\
Al I & $+0.10 \pm 0.03$ & 4 & Ni I & $+0.06 \pm 0.03$ & 81 \\
Si I & $+0.08 \pm 0.03$ & 38 & Zn I & -0.04 & 2 \\
Si II & $+0.06 \pm 0.04$ & 2 & Sr I & +0.09 & 1 \\
S I & -0.02 & 2 & Sr II & +0.10 & 1 \\
Ca I & $+0.14 \pm 0.03$ & 13 & Y II & $+0.10 \pm 0.04$ & 7 \\
Ca II & $+0.16 \pm 0.03$ & 2 & Ba II & +0.27 & 1 \\
Sc II & $+0.15 \pm 0.04$ & 6 & Ce II & +0.06 & 1 \\
Ti I & $+0.08 \pm 0.03$ & 30 & Li I & +1.08 & 1 \\
Ti II & $+0.17 \pm 0.03$ & 9 & & & \\
\hline
\end{tabular}

Notes. The third column lists the number of lines used to determine the mean abundance listed in the second column. The quoted uncertainties are intrinsic RMS errors on the mean value.

\section{CoRoT observations}

The star HD 49385 was one of the targets of the second Long Run in the asteroseismology field of the space mission CoRoT. 136.9 days of photometric data have been collected from October 2007 to March 2008, with a duty cycle of $88.2 \%$. After being corrected from known instrumental effects as described in Samadi et al. (2007a), the lightcurve consists of a series of equally-spaced measurements $(32 \mathrm{~s})$ in the heliocentric frame (also known as N2 data). Small gaps remain in the lightcurve (a few minutes long) mainly due to the passage of the CoRoT satellite in the South Atlantic Anomaly (SAA). A larger gap (3.5 days) was caused by an unexpected reset of the DPU (Digital Processing Unit), which was probably the consequence 


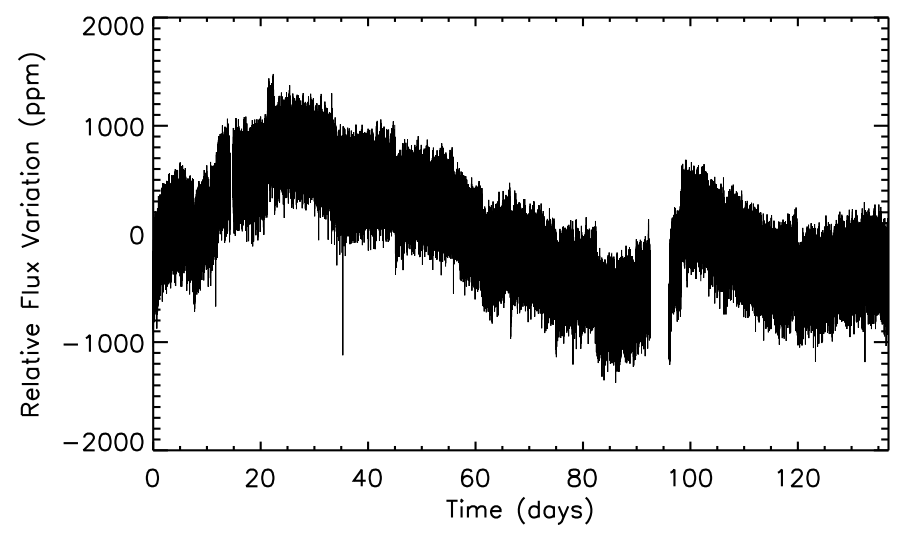

Fig. 4. Lightcurve of 137 days of CoRoT observations on HD 49385, detrended using instrumental information.

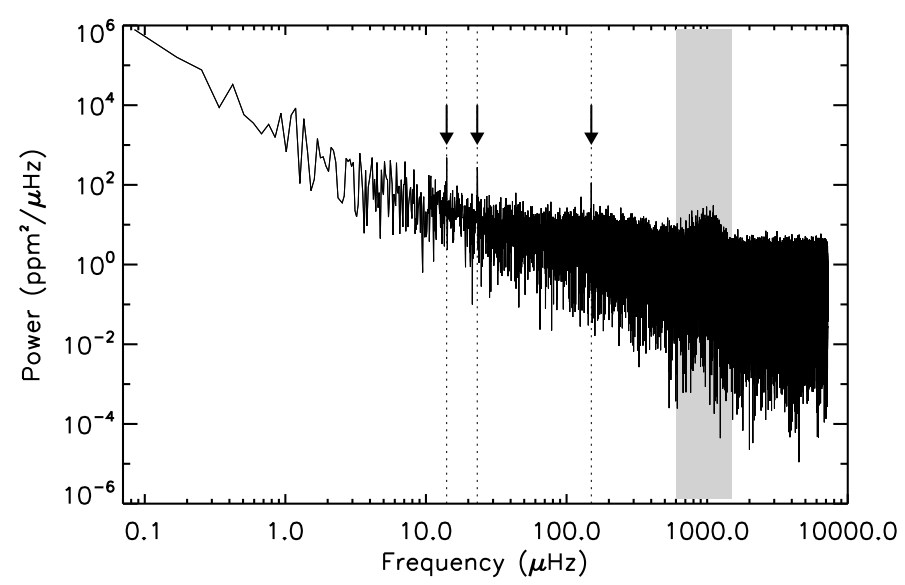

Fig. 5. Power spectrum of 137 days of CoRoT data for HD 49385. The peaks indicated by arrows are from left to right: the $14-\mu \mathrm{Hz}-$ peak discussed in Sect. 4, a peak at $2 v_{\text {day }}=23.1 \mu \mathrm{Hz}$, and a peak at $v_{\text {orb }}-v_{\text {day }}=150.1 \mu \mathrm{Hz}$. The grey area shows the excess of power due to the p-mode oscillations.

of the impact of an energetic particle, because this event occured while the satellite was crossing the SAA. These gaps were linearly interpolated, based on the measurements collected $1000 \mathrm{~s}$ on either sides of the gap. A low-frequency trend due to the aging of the CCD was also corrected by removing a linear gain variation of $-5.3210^{-5}$ day $^{-1}$ (see Auvergne et al. 2009 for more details). The resulting lightcurve is shown in Fig. 4.

Figure 5 shows the power spectrum of the lightcurve, computed with the Fast Fourier Transform algorithm (FFT). It was normalized so that the integrated power spectrum from 0 to twice the Nyquist frequency corresponds to the variance of the time series. At low frequency the background rises due to stellar granulation and possibly to stellar activity (see discussion in Sect. 4). The signature of perturbations due to the orbit of the satellite $\left(v_{\text {orb }} \simeq 161.7 \mu \mathrm{Hz}\right)$ and the day $\left(v_{\text {day }} \simeq 11.6 \mu \mathrm{Hz}\right)$ remains in the power spectrum. These perturbations were expected (see Auvergne et al. 2009) and are of relatively low amplitudes (a few tens of $\mathrm{ppm}^{2} / \mu \mathrm{Hz}$ for the strongest ones at low frequency). They appear as combinations of the harmonics of both frequencies. For instance, a peak appears at $150.1 \mu \mathrm{Hz}$ (corresponding to $v_{\text {orb }}-v_{\text {day }}$ ). Finally, a broad excess of power, centered around $1 \mathrm{mHz}$, is clearly seen in the power spectrum and corresponds to the acoustic modes of HD 49385, which are studied in Sect. 5.

\section{Search for a signature of stellar rotation in the Fourier domain}

The measurement of the rotation period from the lightcurve analysis was found to be useful in previous analyses of p-mode parameters (see Appourchaux et al. 2008; Barban et al. 2009; Mosser et al. 2009b). It indeed provides a direct measurement of the surface rotation period and hence a first estimate of the rotational splitting value.

Contrary to other solar-like pulsating CoRoT targets, no immediate signature of the activity can be seen in the lightcurve (see Fig. 4). Only a long period trend stands out. The same trend was also found in several stars observed on the same CCD during the same specific observation run. Its instrumental or environmental origin is thus established and is currently under study.

We first identified a peak around $0.4 \mu \mathrm{Hz}$ (period of about 29 days), the significance of which is hard to assess because it presents a relatively low amplitude in a frequency domain where the intrumental noise can dominate the power spectrum. We performed a time-frequency analysis with Morlet wavelets (as described in Mathur et al. 2010) which showed that this peak presents a certain stability in time. But because the periods we were testing correspond to a non-negligible fraction of the total observation period, the cone of influence (Torrence \& Compo 1998) prevented us from drawing any conclusion.

A prominent peak can also be detected at a frequency of $14 \mu \mathrm{Hz}$ (period of about 0.8 day) with a signal-to-noise ratio of more than 10 . This peak is however unlikely to be directly related to rotation. Indeed, let us assume ad absurdum that the rotation frequency is $14 \mu \mathrm{Hz}$. Our estimate of the radius implies a surface velocity of more than $120 \mathrm{~km} \mathrm{~s}^{-1}$. To obtain the mea$\operatorname{sured} v \sin i$, we would need a value of the inclination angle lower than $2^{\circ}$, which means that the star is seen pole-on. In this case it is extremely difficult to derive the signature of the activity from the light curve (Mosser et al. 2009a) and no peak related to the rotation period can be detected in the power spectrum, which contradicts our assumption. In the power spectrum of HD 49933 , which is a target of the seismo-field for the same run, we also found a small peak around $14 \mu \mathrm{Hz}$, suggesting this peak might be instrumental. However, the frequencies do not match exactly, and the peak observed for HD 49933 does not appear to be significant. The origin of this peak remains unclear. But it cannot be related to rotation.

In summary, we found no clear signature of the stellar rotation of HD 49385. The low-frequency part of the power spectrum can therefore not be used to obtain a first estimate of the rotational splitting. We may however infer from the absence of a clear activity signal either that the star has a low activity, which cannot be detected even with the CoRoT sensitivity, or that the inclination of the star is close to pole-on.

\section{Analysis of $p$ mode oscillations}

Figure 6 shows the power spectrum smoothed with a $10-\mu \mathrm{Hz}$ boxcar. The comb-like structure of this excess of power is typical of p-mode oscillations. This section presents the analysis of these modes.

\subsection{Frequency of maximum power and large spacing}

To estimate the frequency of the maximum of signal, we fitted a Gaussian profile on the raw power spectrum in the frequency range of the oscillations and above a fitted background (fit described in Sect. 5.3). The result is shown in Fig. 6. We 


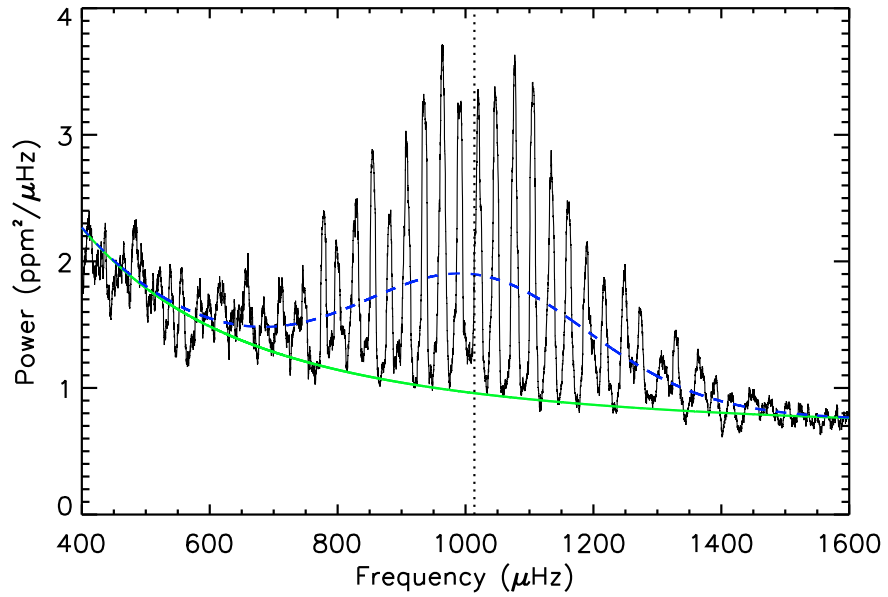

Fig. 6. Smoothed power spectrum of HD 49385 (with a $10 \mu \mathrm{Hz}$ boxcar) in the frequency range of the oscillations. The green curve represents the fitted background (granulation and white noise components). The blue dashed curve corresponds to a Gaussian fit of the raw power spectrum giving $v_{\max }=1013 \mu \mathrm{Hz}$ (dotted line).

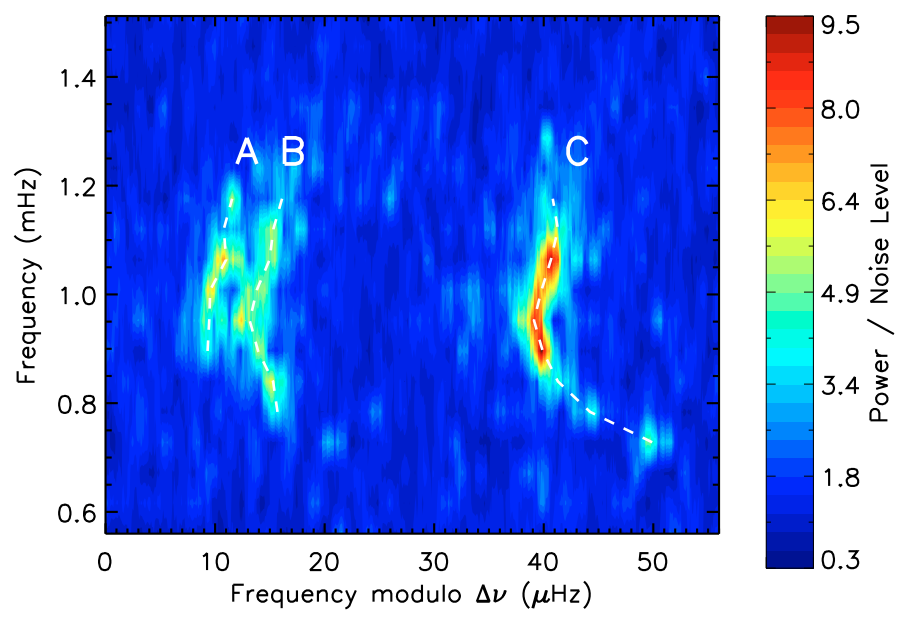

Fig. 7. Échelle diagram of HD 49385 computed with a large spacing of $\Delta v=56 \mu \mathrm{Hz}$. The power spectrum is smoothed with a $1-\mu \mathrm{Hz}$ boxcar and normalized by the background (estimated in Sect. 5.3.1). Three clear ridges appear, labeled here as ridges $\mathrm{A}, \mathrm{B}$, and $\mathrm{C}$. The white dashed lines correspond to the frequencies obtained from a fit to the data presented in Sect. 5.3, and summarized in Tables 3 and 4.

obtained as the frequency of maximum power $v_{\max }=1013 \pm$ $3 \mu \mathrm{Hz}$.

In Fig. 6 a comb-like structure is clearly visible between 600 and $1400 \mu \mathrm{Hz}$. The autocorrelation of the power spectrum provides a first estimate of the mean value of the large spacing $\overline{\Delta v} \simeq 56 \mu \mathrm{Hz}$.

\subsection{Mode identification}

We used the value of the large spacing given in the previous section to build the échelle diagram shown in Fig. 7. It is obtained by piling onto one another sections of $56 \mu \mathrm{Hz}$ of the power spectrum. Three clear ridges appear in the échelle diagram: two neighboring ones, referred to as ridges $\mathrm{A}$ and $\mathrm{B}$ in Fig. 7, and a third one (ridge $\mathrm{C}$ ).

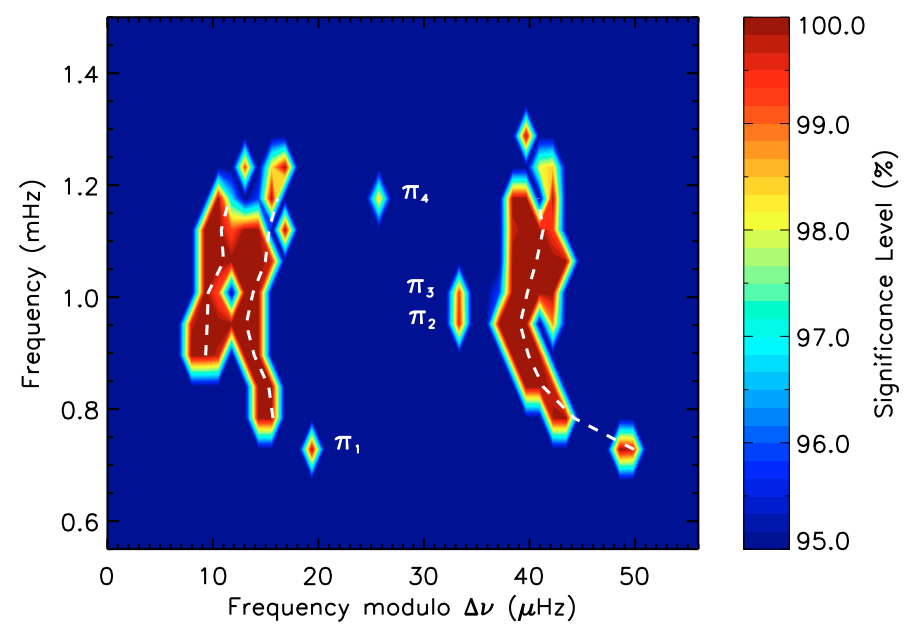

Fig. 8. Échelle diagram of the peaks with a significance greater than $95 \%$ in the power spectrum smoothed over $n=15$ bins $(\sim 1 \mu \mathrm{Hz})$. Four peaks appear, which do not belong to the identified ridges $\mathrm{A}, \mathrm{B}$, or $\mathrm{C}$, and are labeled as $\pi_{1,2,3,4}$.

The pattern we observe in Fig. 7 is the one we expect to observe for low-degree high-radial-order modes in solar-like pulsators: two main ridges separated by about half a large spacing corresponding to $\ell=0$ and $\ell=1$ modes, and alongside the $\ell=0$ ridge a fainter one corresponding to $\ell=2$ modes. It then seems reasonable to identify the ridges as follows: neighboring ridges $\mathrm{A}$ and $\mathrm{B}$ correspond to $\ell=2$ and $\ell=0$ modes, respectively, and ridge $\mathrm{C}$ to $\ell=1$ modes. We note that Mosser \& Appourchaux (2009) and Bedding \& Kjeldsen (2010) obtained the same identification for the degrees of the ridges using different approaches. The following analysis is based on this identification. However, since the previous analyses of CoRoT solar-like targets encountered ambiguity indentifying the modes (see Barban et al. 2009, García et al. 2009), we decided to also consider the possibility of the alternate identification (for which ridge $\mathrm{C}$ corresponds to $\ell=0$ modes), which is discussed in Sect. 5.3.4.

Figure 7 also shows fainter peaks outside the identified ridges. We here study their significance by testing the $\mathrm{H}_{0}$ hypothesis in the frequency range of the oscillations. Because we searched for short-lived modes, we binned the power spectrum over $n$ bins, as prescribed in Appourchaux (2004). The size of the boxcar ( $n=15$ bins $\sim 1 \mu \mathrm{Hz}$ ) for the binning was chosen to approximately match the expected linewidth for the $\mathrm{p}$ modes in solar-like pulsators. In this case, the noise is distributed as a $\chi^{2}$ with $2 n$ degrees of freedom. We then applied the $\mathrm{H}_{0}$ hypothesis, i.e. we supposed that the observations are due to pure noise. For each peak we computed the probability $p$ that pure noise is able to reach the height of the peak over the whole studied window. Low values of $p$ indicate a poor compatibility between the observations and noise, and the significance of the peaks is defined as $1-p$. We show in Fig. 8 all the peaks with a significance higher than $95 \%$.

We notice four peaks which appear with a significance above $98 \%$, and lie outside the identified ridges. They are labeled as $\pi_{1}$, $\pi_{2}, \pi_{3}$ and $\pi_{4}$ on Fig. 8 . The peak $\pi_{4}$, clearly outside the pattern expected from asymptotic theory, could correspond to a mixed mode, given that HD 49385 is an evolved object. The peak $\pi_{1}$ might be an $\ell=0$ mode, assuming that the curvature of ridge $\mathrm{B}$ changes at low frequency. This hypothesis seems to be corroborated by the fact that such a change exists in ridge $C$. However, a more precise inspection shows that including $\pi_{1}$ in ridge $\mathrm{B}$ 
generates an abrupt step in the $\ell=0$ large separation, whereas the $\ell=1$ large separation varies quite smoothly. The peak $\pi_{1}$ could also be an $\ell=1$ mixed mode in avoided crossing. Indeed, at low frequency, the $\ell=0$ and $\ell=1$ ridges have different curvatures. This can be seen in Fig. 7 and will be confirmed by the large separation profiles derived from the fitted mode frequencies (see Sect. 5.3.2). It was shown in Deheuvels \& Michel (2009) that a low-degree $\ell$ avoided crossing creates a characteristic distortion in the ridge of degree $\ell$. If we assume that $\pi_{1}$ is an $\ell=1$ mixed mode in avoided crossing, the expected curvature of the $\ell=1$ ridge is very similar to that of the observed ridge. We cannot establish this identification more firmly at this stage though. Because of the uncertainty regarding the identification of the $\pi_{1}$ mode, we preferred to consider and fit it individually (see Sect. 5.3). The peaks $\pi_{2}$ and $\pi_{3}$ are located at about $7 \mu \mathrm{Hz}$ on the left of ridge $\mathrm{C}$. We studied the possibility for these peaks to correspond to mixed modes. In this case, they would have to be of different degree $\ell$. Indeed, g modes of same degree and increasing radial order have frequencies increasingly close to each other. Two mixed modes of same degree $\ell$ spaced by $\Delta v$ would imply many more mixed modes in the ridge of degree $\ell$ at lower frequency. It is clear from ridges $\ell=1$ and $\ell=2$ that this is not the case. And if $\pi_{2}$ and $\pi_{3}$ were mixed modes of different degree, we would expect them to have different amplitudes, which does not seem to be the case. Their position in the échelle diagram on the left of ridge $\mathrm{C}$ suggests that they could also be $\ell=3$ modes. Several reasons led us to favor this identification. First, these two modes appear vertically aligned in the échelle diagram and around the maximum of the oscillations, i.e. at the place where it is most likely to observe $\ell=3$ modes if they are present. Then, $\pi_{2}$ and $\pi_{3}$ might be part of a fainter ridge (referred to as ridge $\mathrm{D}$ ), because other peaks, which have a lower significance but seem to follow the alignment, appear in Fig. 7. Finally, the distance between ridges $\mathrm{C}$ and $\mathrm{D}$ is also consistent with this identification. Indeed, the asymptotic theory gives an expression for the average distance between the ridges

$\left\langle\delta v_{n, \ell}\right\rangle \equiv v_{n, \ell}-v_{n-1, \ell+2}=(4 \ell+6) D_{0}$,

where the expression of $D_{0}$ is found in Christensen-Dalsgaard (2003). By definition, the quantity $\left\langle\delta v_{n, \ell=0}\right\rangle$ corresponds to the distance between the $\ell=0$ and $\ell=2$ ridges, and $\left\langle\delta v_{n, \ell=1}\right\rangle$ corresponds to the distance between the $\ell=1$ and $\ell=3$ ridges. Figure 7 shows that we have $\left\langle\delta v_{n, \ell=0}\right\rangle \simeq 5 \mu \mathrm{Hz}$. We therefore expect $\ell=3$ modes to be approximately $8 \mu \mathrm{Hz}$ on the left of $\ell=1$ modes, which approximately matches the position of ridge D.

The significance is an efficient tool to select peaks, but it is not a guarantee for the detection of a signal, as stressed in Appourchaux et al. (2009). To confirm the existence of a signal, one needs to compute the posterior probability $P\left(H_{0} \mid X=x\right)$. The details of the calculation along with the assumptions we make are given in Appendix B. For $\pi_{1}$ and $\pi_{4}$, assumed to be mixed modes, we found posterior probabilities of $0.001 \%$ and $8.7 \%$, respectively. These peaks are therefore probably due to signal. The larger posterior probability found for $\pi_{4}$ is caused by the fact that the expected profile of mixed modes was built with very conservative priors . For $\pi_{2}$ and $\pi_{3}$, assumed to correspond to $\ell=$ 3 modes, we obtained a very low posterior probability $(1.4 \%$ and $1.1 \%$, respectively), indicating that these peaks are highly likely due to signal, and a good compatibility between their observed profiles and the expected profile of $\ell=3$ modes for HD 49385 . This confirms our identification for $\pi_{2}$ and $\pi_{3}$. It is the first time that $\ell=3$ modes can be detected in a solar-like pulsator (other than the Sun). We explain in Sect. 5.3.2 why HD 49385 was a particularly favorable target to detect $\ell=3$ modes.
We remark that ridge $\mathrm{D}$ could also be seen as the $m=+1$ component of the rotationally splitted $\ell=1$ mode. This hypothesis is studied in Sect. 5.3.4.

\subsection{Extraction of $p$ mode parameters}

\subsubsection{Method}

Solar-like oscillations are the result of stochastic excitations of $p$ modes in the convective envelope. They respond to the equation of a forced, damped and randomly excitated harmonic oscillator (see Duvall \& Harvey 1986). Therefore their signature in the power spectrum can be modeled as Lorentzian profiles with a linewidth proportional to the inverse of the mode lifetimes, added to the background and perturbated by a multiplicative noise following a $\chi^{2}$ distribution with two degrees of freedom. We used a maximum likelihood estimation (MLE) to determine the most probable parameters of the observed p modes (see Anderson et al. 1990). This method was already applied to determine p-mode parameters for the Sun (Toutain \& Froehlich 1992; Appourchaux et al. 1998) as well as for CoRoT solar-like targets (Appourchaux et al. 2008; Barban et al. 2009; García et al. 2009). As described in Anderson et al. (1990), the likelihood $L$ is defined as the product of the probability density of each frequency bin $v_{i}$ of the power spectrum

$$
L\left(v_{i}, \lambda\right)=\prod_{i} \frac{1}{M\left(v_{i}, \lambda\right)} \exp \left(\frac{-S\left(v_{i}\right)}{M\left(v_{i}, \lambda\right)}\right),
$$

where $S$ is the observed power spectrum, $M$ the model described above, and $\lambda$ the parameters of the Lorentzian profiles used in the model. The set of parameters $\lambda$ that maximizes the likelihood corresponds to the most probable parameters. Maximizing $L$ is the same as minimizing the negative logarithmic likelihood $\mathcal{L}$, defined as

$$
\mathcal{L} \equiv-\ln (L)=\sum_{i}\left(\ln M\left(v_{i}, \lambda\right)+\frac{S\left(v_{i}\right)}{M\left(v_{i}, \lambda\right)}\right) .
$$

As mentioned above, perturbations remain in the power spectrum at frequencies corresponding to combinations of the harmonics of the orbital frequency and those of the day. Some of these perturbations can be seen in the frequency domain of the oscillations. We therefore gave a null weight to the corresponding bins so that our estimates of the p-mode parameters are not influenced by those peaks.

We estimated the background with a power law to describe the granulation component, as prescribed by Harvey (1985). Prior to the fit of the p-mode parameters, we fitted a profile of the type

$$
B\left(v_{i}\right)=\frac{\alpha}{1+\left(\beta v_{i}\right)^{\gamma}}+\delta
$$

on the power spectrum, excluding the frequency domain of the p-mode oscillations to avoid any bias, with $\alpha, \beta, \gamma$ and $\delta$ as free parameters. We then used this background to fit the mode parameters.

The analysis of HD 49933, which was the first solar-like CoRoT target, showed that a global fit of all the modes gave more robust estimates of the mode parameters (see Appourchaux et al. 2008). We therefore fitted modes simultaneously on a frequency range corresponding to nine overtones in the power spectrum. The assumptions made for the fit are the same as in Appourchaux et al. (2008). We briefly recall them here. The splitting was assumed to be constant over the frequency range of 


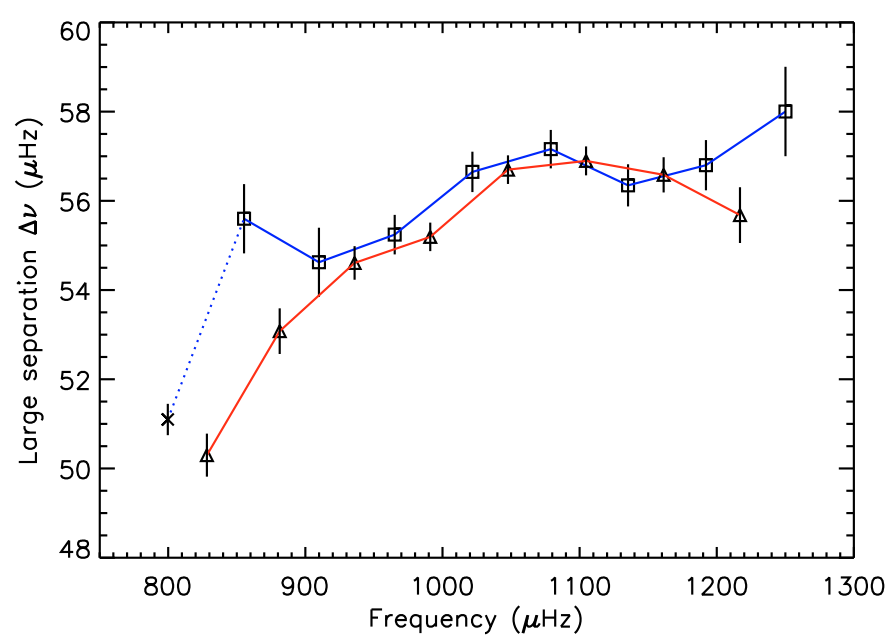

Fig. 9. Large separation profiles derived from the fitted frequencies of the $\ell=0$ (squares and blue line) and $\ell=1$ (triangles and red line) modes. We overplotted the 1- $\sigma$ error bars. The cross corresponds to the large separation we would obtain, if $\pi_{1}$ were considered as an $\ell=0$ mode.

the oscillations, and the $m \neq 0$ components of non-radial modes were supposed to be symmetrical with respect to the $m=0$ component. We sliced the power spectrum in $\Delta v$-wide intervals, in which the linewidth is assumed to be the same for all modes, and where only the height of the $\ell=0$ mode $\left(H_{\ell=0, n}\right)$ is fitted. The heights of non-radial modes are determined from those of the $\ell=0$ mode. Assuming an equal repartition of the energy in the modes of different degrees $\ell$, and taking the value of the limb darkening for this star into account, we obtain

$H_{\ell=1, n} / H_{\ell=0, n}=1.5$

$H_{\ell=2, n} / H_{\ell=0, n}=0.5$

$H_{\ell=3, n} / H_{\ell=0, n}=0.05$,

where $H_{\ell=1, n}=\sum_{m=-\ell}^{m=+\ell} H_{n, \ell, m}$. Inside non-radial multiplets, the heights $H_{n, \ell, m}$ are given by the intensity visibilities (see Gizon \& Solanki 2003; Ballot et al. 2006).

\subsubsection{Mode parameters estimates}

Five teams worked independently on the extraction of the pmode parameters and applied the method we described in Sect. 5.3.1 to fit the four ridges associated to $\ell=0,1,2,3$ modes. As was done in previous analyses of this type, we present in the following section one set of reference results, and we use results from the other teams to cross-check them and comment the robustness of the solution. The reference results of the fit are given in Tables 3 and 4.

\section{Mode frequencies}

There is a very good agreement between the different teams on the fitted mode eigenfrequencies. Only two frequencies in the $\ell=3$ ridge differ by more than $1 \sigma$. Indeed, this ridge is too faint to offer reliable estimates of the mode frequencies away from the maximum of the signal. An échelle diagram of the fitted modes is given in Fig. 10. At the maximum of the signal, the obtained precision on the mode frequencies ranges from about 0.2 to about $0.6 \mu \mathrm{Hz}$ for $0 \leqslant \ell \leqslant 3$ modes and increases at the edges of the frequency domain of the oscillations.

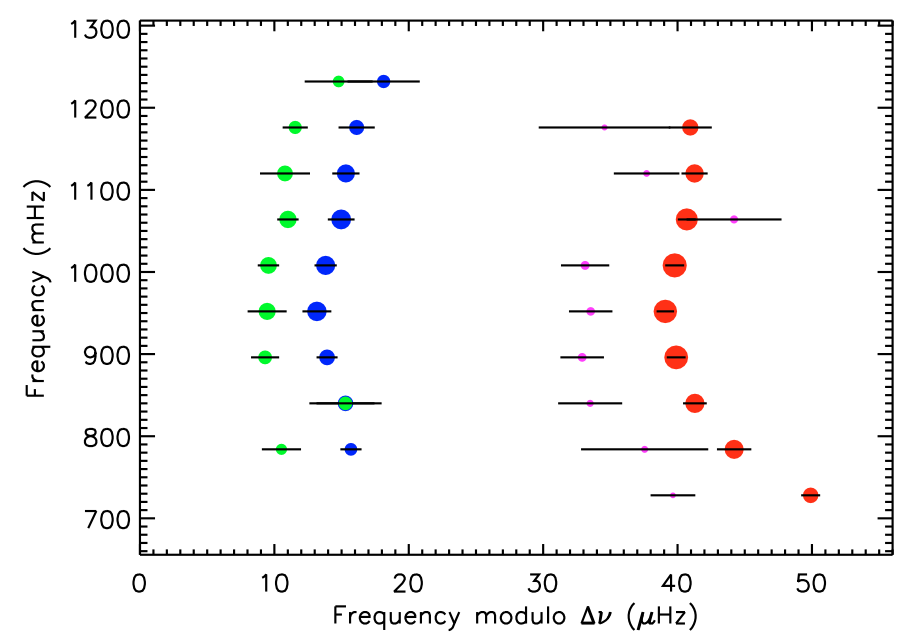

Fig. 10. Échelle diagram of the fitted modes. The colors correspond to the following degrees: blue $\ell=0$, red $\ell=1$, green $\ell=2$ and purple $\ell=3$. The surface of the disks is proportional to the heights of the modes. 3- $\sigma$ error bars are overplotted.

At $v=855.3 \mu \mathrm{Hz}$, the $\ell=2$ mode is found merged together with the $\ell=0$ mode. This could be because the signal-to-noise ratio decreases when reaching the edges of the fitted domain. Indeed, with a lower signal-to-noise ratio, the analysis of previous CoRoT targets yielded several $\ell=2$ modes merged with the neighboring $\ell=0$ mode (see Appourchaux et al. 2008; Barban et al. 2009). However, the $\ell=2$ mode might also have been shifted because of an $\ell=2$ mixed mode in the neighborhood.

Figure 9 shows the large separation profiles derived from the fitted frequencies of the $\ell=0$ and $\ell=1$ modes. It appears, as mentioned in Sect. 5.2, that the $\ell=0$ and $\ell=1$ ridges have a different curvature at low frequency, which might be caused by a low-frequency avoided crossing.

\section{Mode linewidths}

Figure 11 shows the linewidths of the fitted modes. As was observed in the Sun, the linewidths of the modes increase with frequency and are almost constant around the maximum of the signal. They are larger than those of the Sun by a factor 2 . The lifetimes $\tau$ of the modes can be deduced from the linewidth by the relation $\tau=1 /(\pi \Gamma)$. For HD 49385 they range from about one day to two days. By comparison the mode lifetimes are shorter in other CoRoT solar-like pulsators, e.g. for HD 181420 (about six hours for the shortest ones, and 12 hours around the maximum of the signal).

It appears that one of the fitted intervals $(v=855.3 \mu \mathrm{Hz}$ for the $\ell=0$ mode) has a linewidth somewhat larger than what could be expected considering the linewidths of neighboring orders (see Fig. 11). Indeed, the $\ell=1$ mode of this interval $(v=828.2 \mu \mathrm{Hz})$ is larger than the other modes in the neighborhood.

\section{Mode heights and amplitudes}

As was mentioned before, we fixed the ratios of heights beween modes of different degrees $\ell$ in our fit. Because HD 49385 is the first CoRoT target for which the $\ell=0$ and $\ell=2$ ridges are 
Table 3. Reference results for the parameters of the radial $\mathrm{p}$ modes.

\begin{tabular}{cccccccccc}
\hline \hline$\ell$ & $v_{n, \ell}(\mu \mathrm{Hz})$ & agr. $\left(v_{n, \ell}\right)$ & $H_{0, n}\left(\mathrm{ppm}^{2} / \mu \mathrm{Hz}\right)$ & agr. $\left(H_{0, n}\right)$ & $\Gamma_{n}(\mu \mathrm{Hz})$ & agr. $\left(\Gamma_{n}\right)$ & $a_{n}(\mathrm{ppm})$ & agr. $\left(a_{n}\right)$ & $H_{\ell, n} / B\left(v_{n, \ell}\right)$ \\
\hline 0 & $799.70 \pm 0.27$ & $\bullet$ & $2.55_{-0.61}^{+0.80}$ & $\bullet$ & $1.58_{-0.33}^{+0.42}$ & $\bullet$ & $3.55_{-0.56}^{+0.67}$ & $\bullet$ & 2.23 \\
0 & $855.30 \pm 0.73$ & $\bullet$ & $2.10_{-0.46}^{+0.59}$ & $\bullet$ & $2.97_{-0.58}^{+0.72}$ & $\bullet$ & $4.42_{-0.65}^{+0.77}$ & $\bullet$ & 1.94 \\
0 & $909.92 \pm 0.26$ & $\bullet$ & $3.13_{-0.65}^{+0.82}$ & $\bullet$ & $2.00_{-0.35}^{+0.43}$ & $\bullet$ & $4.44_{-0.60}^{+0.770}$ & $\bullet$ & 3.03 \\
0 & $965.16 \pm 0.36$ & $\bullet$ & $4.05_{-0.78}^{+0.96}$ & $\bullet$ & $2.37_{-0.37}^{+0.43}$ & $\bullet$ & $5.49_{-0.72}^{+0.83}$ & $\bullet$ & 4.09 \\
0 & $1021.81 \pm 0.28$ & $\bullet$ & $4.56_{-0.92}^{+1.15}$ & $\bullet$ & $2.03_{-0.34}^{+0.41}$ & $\bullet$ & $5.40_{-0.70}^{+0.80}$ & $\bullet$ & 4.78 \\
0 & $1078.97 \pm 0.33$ & $\bullet$ & $4.77_{-0.90}^{+1.10}$ & $\bullet$ & $2.08_{-0.30}^{+0.35}$ & $\bullet$ & $5.58_{-0.70}^{+0.80}$ & $\bullet$ & 5.18 \\
0 & $1135.32 \pm 0.34$ & $\bullet$ & $3.49_{-0.67}^{+0.83}$ & $\bullet$ & $2.37_{-0.37}^{+0.44}$ & $\bullet$ & $5.09_{-0.73}^{+0.86}$ & $\bullet$ & 3.90 \\
0 & $1192.12 \pm 0.45$ & $\bullet$ & $2.29_{-0.52}^{+0.67}$ & $\bullet$ & $2.51_{-0.53}^{+0.68}$ & $1.8 \sigma$ & $4.25_{-0.67}^{+0.80}$ & $\bullet$ & 2.63 \\
0 & $1250.12 \pm 0.89$ & $\bullet$ & $1.15_{-0.25}^{+0.32}$ & $\bullet$ & $3.90_{-0.80}^{+1.00}$ & $\bullet$ & $3.76_{-1.95}^{+.04}$ & $\bullet$ & 1.36 \\
\hline
\end{tabular}

Notes. The heights given here are those of the $\ell=0$ modes (the heights of non-radial modes are computed using height ratios, see Sect. 5.3.1). The agreement (agr.) between the teams is given for each fitted parameter. Filled circles indicate that at least four of the five teams agreed on the value within $1 \sigma . X \sigma$ indicates that more than one team disagrees with the value, and that the five teams agree within $X \sigma(X>1)$.

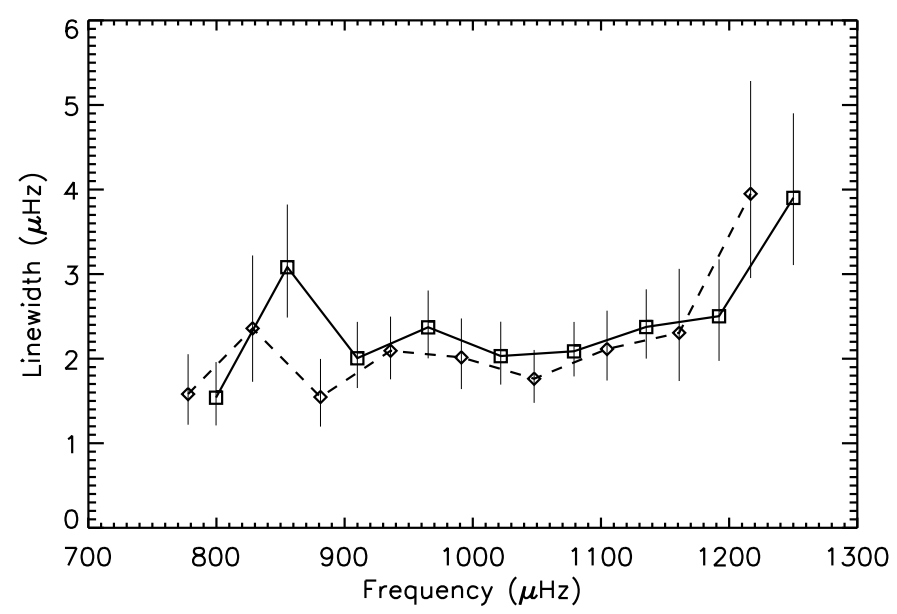

Fig. 11. Linewidths of the fitted modes with 1- $\sigma$ error bars (squares and full line). The linewidths for the alternate identification are also given (diamonds and dashed line).

clearly distinct in the échelle diagram, we had the opportunity to check the chosen height ratios, which are those usually adopted in this type of analysis. We performed a new fit releasing the height ratios and found

$H_{\ell=1, n} / H_{\ell=0, n}=1.54 \pm 0.16$

$H_{\ell=2, n} / H_{\ell=0, n}=0.76 \pm 0.12$

$H_{\ell=3, n} / H_{\ell=0, n}=0.20 \pm 0.05$.

For $\ell=1$ modes, the theoretical value of the ratio lies within the $1-\sigma$ error bars of the fitted value. For $\ell=2$ and $\ell=3$ modes, the theoretical ratios are at 2 and $3 \sigma$ of the fitted values, respectively. At this stage, this difference is too small to be interpreted as an inconsistency with the theoretical height ratios. It would be interesting however to investigate this matter in future analyses of this type on different objects. We noticed that with free height ratios the results for mode frequencies, linewidths and amplitudes remained within the $1-\sigma$ error bars of the parameters obtained with fixed height ratios.

From the heights and linewidths of the modes we derived the amplitudes (in ppm), given by $A=\sqrt{\pi H \Gamma}$. To estimate the error bars on this quantity, we took into account the anticorrelation which exists between the determination of the height and the linewidth. The mode amplitudes are represented for $\ell=0$ modes as a function of frequency in Fig. 12. They range from $3.6 \mathrm{ppm}$ to $5.4 \mathrm{ppm}$. However, these estimates correspond to instrumental values. Taking into account the instrumental response functions of CoRoT (as described in Michel et al. 2009), they can be converted into intrinsic bolometric amplitudes per radial mode by multiplying them by a factor 1.032 . The obtained amplitudes are consistent with the prediction of the maximum amplitudes using scaling laws $\left(A_{\ell=0}=6.1 \pm 0.5\right.$, see Appendix A). The star HD 49385 is then quite specific among the other solar-like pulsators observed with CoRoT for which amplitudes were found significantly lower than the predicted ones (see Appourchaux et al. 2008; Barban et al. 2009; García et al. 2009; Michel et al. 2008).

The signal-to-noise (SNR) ratio of each mode computed from the fitted height and the background estimated in Sect. 5.3.1 is given in Table 3. The SNR is found to be very small for the fitted $\ell=3$ modes (about 0.25 at the maximum of the signal). However, when releasing the height ratios, we find $H_{\ell=3, n} / H_{\ell=0, n}=0.20 \pm 0.05$. The SNR of the $\ell=3$ modes should be closer to 1 at the maximum of the signal.

\section{Splitting and inclination angle estimates}

Most of the fits we performed converge toward a null value of the splitting and consequently an undefined value of the inclination angle. This can correspond to two different cases:

- The star could be seen almost pole-on, i.e. with an inclination angle close to $0^{\circ}$. In that case the $m \neq 0$ components of the non-radial modes are not visible, and we therefore cannot estimate the splitting. However, the measured $v \sin i$ of the object and the estimate of the stellar radius $R$ allow us to give constraints on the splitting $v_{\mathrm{s}}$ through the relation

$v \sin i=2 \pi R v_{\mathrm{s}} \sin i$.

For a low inclination angle we expect higher values of the rotational splitting (e.g. $v_{\mathrm{s}}>1.5 \mu \mathrm{Hz}$ for $i$ lower than $10^{\circ}$ ).

- We could also explain this result by a low value of the splitting compared to the linewidth of the modes, so that the 


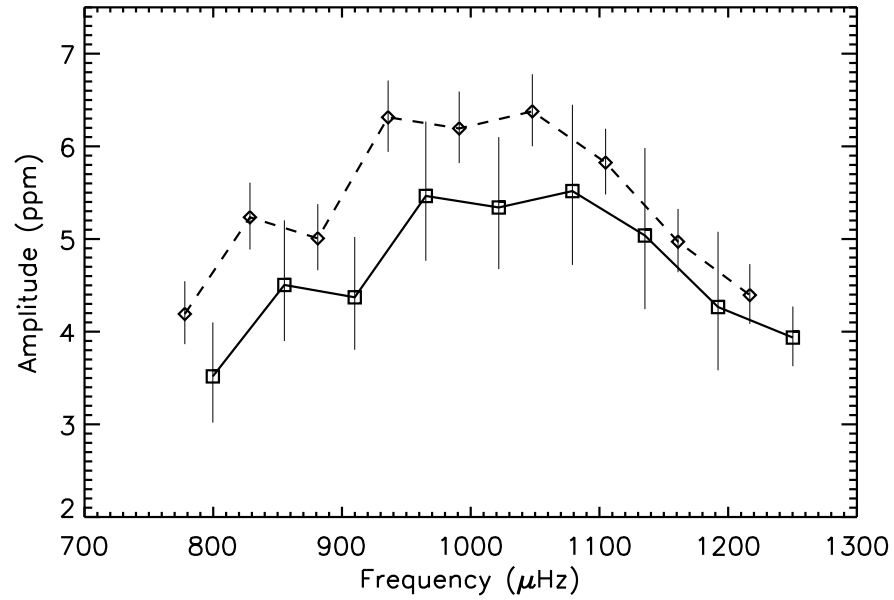

Fig. 12. Amplitudes of the fitted modes with 1- $\sigma$ error bars (squares and full line). The amplitudes for the alternate identification also appear (diamonds and dashed line).

Table 4. Reference results for the parameters of the non-radial $\mathrm{p}$ modes (symbols are the same as in Table 3).

\begin{tabular}{cccc}
\hline \hline$\ell$ & $v_{n, \ell}(\mu \mathrm{Hz})$ & agr. $\left(v_{n, \ell}\right)$ & $H_{\ell, n} / B\left(v_{n, \ell}\right)$ \\
\hline 1 & $777.91 \pm 0.24$ & $\bullet$ & 3.26 \\
1 & $828.21 \pm 0.42$ & $\bullet$ & 2.83 \\
1 & $881.29 \pm 0.29$ & $\bullet$ & 4.44 \\
1 & $935.90 \pm 0.23$ & $\bullet$ & 6.00 \\
1 & $991.09 \pm 0.22$ & $\bullet$ & 7.03 \\
1 & $1047.79 \pm 0.24$ & $\bullet$ & 7.62 \\
1 & $1104.68 \pm 0.22$ & $\bullet$ & 5.76 \\
1 & $1161.27 \pm 0.33$ & $\bullet$ & 3.89 \\
1 & $1216.95 \pm 0.53$ & $\bullet$ & 2.01 \\
\hline 2 & $794.55 \pm 0.52$ & $\bullet$ & 1.11 \\
2 & $855.29 \pm 0.93$ & $\bullet$ & 0.97 \\
2 & $905.31 \pm 0.35$ & $\bullet$ & 1.51 \\
2 & $961.47 \pm 0.49$ & $\bullet$ & 2.04 \\
2 & $1017.56 \pm 0.27$ & $\bullet$ & 2.38 \\
2 & $1075.01 \pm 0.27$ & $\bullet$ & 2.58 \\
2 & $1130.79 \pm 0.61$ & $\bullet$ & 1.95 \\
2 & $1187.55 \pm 0.32$ & $\bullet$ & 1.31 \\
2 & $1246.78 \pm 0.84$ & $\bullet$ & 0.68 \\
\hline 3 & $767.74 \pm 0.65$ & $7.3 \sigma$ & 0.11 \\
3 & $821.55 \pm 1.57$ & $\bullet$ & 0.09 \\
3 & $873.46 \pm 0.83$ & $\bullet$ & 0.15 \\
3 & $928.93 \pm 0.57$ & $\bullet$ & 0.20 \\
3 & $985.53 \pm 0.55$ & $\bullet$ & 0.23 \\
3 & $1041.11 \pm 0.59$ & $\bullet$ & 0.25 \\
3 & $1108.11 \pm 1.33$ & $3.1 \sigma$ & 0.19 \\
3 & $1157.70 \pm 0.83$ & $\bullet$ & 0.13 \\
3 & $1210.55 \pm 1.68$ & $\bullet$ & 0.07 \\
\hline & & &
\end{tabular}

fits are unable to separate the components of the non-radial modes. Equation 5 shows that a small rotational splitting corresponds to a high inclination angle.

This fitting procedure does not significantly favor any of these two hypotheses. However, they lead to almost identical p mode parameters (all the parameters agree within 1- $\sigma$ error bars).
Table 5. Results of a Lorentzian fit performed on the peaks identified as potential mixed modes.

\begin{tabular}{cccc}
\hline \hline$v(\mu \mathrm{Hz})$ & $H\left(\mathrm{ppm}^{2} / \mu \mathrm{Hz}\right)$ & $\Gamma(\mu \mathrm{Hz})$ & $a(\mathrm{ppm})$ \\
\hline $748.60 \pm 0.23$ & $2.82_{-1.20}^{+2.08}$ & $1.12_{-0.46}^{+0.79}$ & $3.15_{-0.92}^{+1.32}$ \\
$1201.96 \pm 0.23$ & $2.03_{-1.02}^{+2.05}$ & $0.67_{-0.31}^{+0.57}$ & $2.07_{-0.70}^{+1.06}$ \\
\hline
\end{tabular}

In both cases, the effect of the rotational splitting on the observed mode profiles is weak. Indeed, either the angle is small, and the $m \neq 0$ components of the multiplets have very small amplitudes compared to the $m=0$ component, or the splitting is small, and the $m$ components are mixed together. We note that this favors the detection of lower degree modes and therefore justifies why $\ell=3$ modes seem to be detected in HD 49385, while they were not in previously analyzed CoRoT targets.

\subsubsection{Possible mixed modes parameter estimates}

The position of HD 49385 in the HR diagram suggests that it either reaches the end of the main sequence or lies shortly after it. The star HD 49385 is therefore evolved and its acoustic spectrum may contain mixed modes, i.e. modes which have a gmode behavior in the deep interior and a p-mode behavior below the surface (see Scuflaire 1974). The mixed modes are known to experience the so-called avoided crossing phenomenon, during which their frequency does not follow the asymptotic approximation and they can be located out of the ridges (Unno et al. 1989). It was already noticed (e.g. Dziembowski \& Pamyatnykh 1991) that the detection of these modes could yield a very valuable constraint on the size of the convective core.

As was noticed in Sect. 5.1, two peaks are detected in the power spectrum with a confidence level above $95 \%$ and are not located within any of the observed ridges in the échelle diagram (namely $\pi_{1}$ and $\pi_{4}$ ). They could be the signature of mixed modes. We note that if the peak $\pi_{1}$, found at $v \sim 748 \mu \mathrm{Hz}$ is an $\ell=1$ mixed mode in avoided crossing, it could explain why the $\ell=1$ p mode at $v \sim 797.5 \mu \mathrm{Hz}$ seems not to follow the curvature of the $\ell=1$ ridge. We fitted these peaks as Lorentzian profiles with free frequencies, heights and linewidths. Because the mixed modes have a g-mode character near the center, their inertia is larger, and we expect them to have a longer lifetime and therefore a smaller linewidth than regular modes. We obtained the results given in Table 5. We notice that the fitted frequencies have small error bars, but the other parameters are ill-determined.

\subsubsection{Other mode identification scenarii}

Can ridge $\mathrm{D}$ be the $m=+1$ component of the $\ell=1$ modes?

Still considering the identification preferred in this paper (i.e. ridge $\mathrm{C}$ corresponding to $\ell=1$ modes), ridge $\mathrm{D}$ could also possibly be seen as the $m=+1$ of the $\ell=1$ ridge, as already mentioned in Sect. 5.1. When not including the $\ell=3$ modes in the fit, we indeed find solutions with a splitting of about $7 \mu \mathrm{Hz}$, corresponding to the distance between ridges $\mathrm{C}$ and $\mathrm{D}$.

However, we ran models representative of HD 49385 and found that with this rotation frequency the $m \neq 0$ components of non-radial multiplets are no longer symmetric with respect to the $m=0$ component. To treat this case correctly, we therefore need to take this asymmetry into account in our fits. 
A\&A 515, A87 (2010)

Table 6. Reference results for the parameters of the radial p modes for the alternate identification (symbols are the same as in Table 3 ).

\begin{tabular}{cccccccccc}
\hline \hline$\ell$ & $v_{n, \ell}(\mu \mathrm{Hz})$ & agr. $\left(v_{n, \ell}\right)$ & $H_{0, n}\left(\mathrm{ppm}^{2} / \mu \mathrm{Hz}\right)$ & agr. $\left(H_{0, n}\right)$ & $\Gamma_{n}(\mu \mathrm{Hz})$ & agr. $\left(\Gamma_{n}\right)$ & $a_{n}(\mathrm{ppm})$ & agr. $\left(a_{n}\right)$ & $H_{\ell, n} / B\left(v_{n, \ell}\right)$ \\
\hline 0 & $777.89 \pm 0.24$ & $\bullet$ & $3.23_{-0.78}^{+1.02}$ & $\bullet$ & $1.58_{-0.36}^{+0.47}$ & $\bullet$ & $4.00_{-0.29}^{+0.31}$ & $\bullet$ & 2.76 \\
0 & $828.22 \pm 0.42$ & $\bullet$ & $3.17_{-0.85}^{+1.16}$ & $\bullet$ & $2.36_{-0.63}^{+0.86}$ & $\bullet$ & $4.85_{-0.28}^{+0.29}$ & $1.4 \sigma$ & 2.85 \\
0 & $881.18 \pm 0.34$ & $\bullet$ & $4.53_{-1.08}^{+1.42}$ & $\bullet$ & $1.55_{-0.35}^{+0.45}$ & $\bullet$ & $4.69_{-0.27}^{+0.29}$ & $\bullet$ & 4.28 \\
0 & $935.83 \pm 0.22$ & $\bullet$ & $5.32_{-0.96}^{+1.17}$ & $\bullet$ & $2.09_{-0.34}^{+0.40}$ & $\bullet$ & $5.92_{-0.27}^{+0.28}$ & $\bullet$ & 5.26 \\
0 & $991.09 \pm 0.25$ & $\bullet$ & $5.32_{-1.05}^{+1.30}$ & $\bullet$ & $2.01_{-0.37}^{+0.46}$ & $\bullet$ & $5.80_{-0.28}^{+0.29}$ & $\bullet$ & 5.47 \\
0 & $1047.86 \pm 0.29$ & $\bullet$ & $6.45_{-1.23}^{+1.52}$ & $\bullet$ & $1.76_{-0.29}^{+0.34}$ & $\bullet$ & $5.98_{-0.27}^{+0.29}$ & $\bullet$ & 6.88 \\
0 & $1104.74 \pm 0.22$ & $\bullet$ & $4.49_{-0.86}^{+1.06}$ & $\bullet$ & $2.11_{-0.37}^{+0.45}$ & $\bullet$ & $5.46_{-0.26}^{+0.27}$ & $\bullet$ & 4.95 \\
0 & $1161.01 \pm 0.34$ & $\bullet$ & $2.99_{-0.73}^{+0.96}$ & $\bullet$ & $2.31_{-0.57}^{+0.76}$ & $\bullet$ & $4.65_{-0.26}^{+0.27}$ & $\bullet$ & 3.38 \\
0 & $1216.77 \pm 0.69$ & $\bullet$ & $1.38_{-0.32}^{+0.42}$ & $\bullet$ & $3.95_{-1.00}^{+1.33}$ & $\bullet$ & $4.14_{-0.26}^{+0.28}$ & $\bullet$ & 1.60 \\
\hline
\end{tabular}

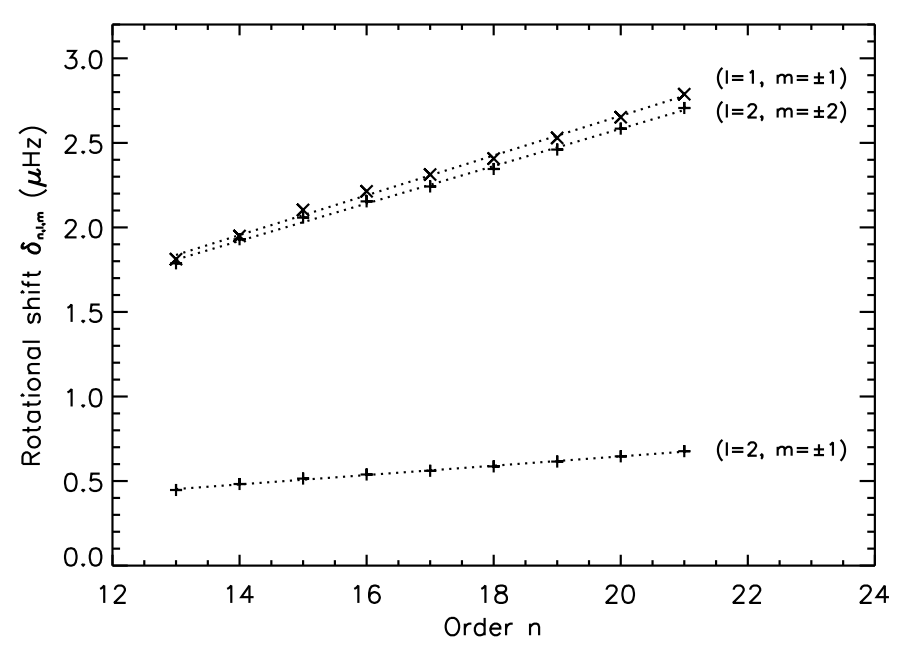

Fig. 13. Frequency shift $\delta_{n, \ell, m}$ (defined in Eq. (6)) of the $m \neq 0$ components of non-radial multiplets for an evolutionary model representative of HD 49385 in the frequency range of the detected oscillations and with a splitting of $3 \mu \mathrm{Hz}$. Crosses stand for $\ell=1$ modes and plus signs for $\ell=2$ modes. A linear regression of the variations of the shifts with respect to the frequency is overplotted (dotted lines).

For this purpose, we studied this asymmetry on evolutionary models reproducing the $T_{\text {eff }}$ and $L$ that we obtained for HD 49385 in Sect. 2.1 and with different angular velocities. The models were computed with the evolutionary code CESAM2k (Morel 1997) and the eigenfrequencies were derived from them with the oscillation code FILOU (Suárez \& Goupil 2008). They showed that the effects of asymmetry should be non-negligible even for values of the splitting as low as a few $\mu \mathrm{Hz}$. We describe the asymmetry of the $m \neq 0$ components of a mode of degree $\ell$ and order $n$ by the shift $\delta_{n, \ell, m}$ defined as

$\delta_{n, \ell, m} \equiv v_{n, \ell, 0}-\frac{v_{n, \ell,+m}+v_{n, \ell,-m}}{2}$.

These shifts are represented in Fig. 13 for a model representative of HD 49385 for eigenmodes in the frequency range of the detected oscillations $(13 \leqslant n \leqslant 21)$ and with a rotational splitting of $3 \mu \mathrm{Hz}$. We observe that the shifts $\delta_{n, \ell, m}$ are expected to vary approximately linearly with respect to frequency. We therefore model the shift $\delta_{n, \ell, m}$ as

$\delta_{n, \ell, m}=\alpha_{\ell, m}\left(n-n_{0}\right)+\delta_{n_{0}, \ell, m}$, where $n_{0}$ corresponds to the order where the signal is maximal $\left(n_{0}=17\right)$. For $m \neq 0$ modes in non-radial multiplets we now fit eigenfrequencies of the type

$v_{n, \ell, m}=v_{n, \ell, 0}+m v_{\mathrm{s}}-\delta_{n, \ell, m}$.

This adds two free parameters to the fit per fitted $m \neq 0$ component.

The obtained results for the eigenfrequencies, heights, linewidths and amplitudes of modes of degrees $0 \leqslant \ell \leqslant 2$ all lie within the 1- $\sigma$ error bars of those given in Table 3 . The fit converges toward a splitting of $v_{\mathrm{s}}=3.8 \pm 0.2 \mu \mathrm{Hz}$ and an angle of $i=20.5 \pm 2.3^{\circ}$. As expected, ridge $\mathrm{D}$ is fitted as the $m=+1$ component of the $\ell=1$ modes. The obtained rotational shift is such that $\alpha_{\ell=1, m=1}=0.30 \pm 0.19 \mu \mathrm{Hz}$ per order $n$, and $\delta_{n_{0}, \ell=1, m=1}=3.0 \pm 0.6 \mu \mathrm{Hz}$. However, using Eq. (5) and the fitted values of the splitting and the angle, we obtain $v \sin i=11.2 \pm 2.8 \mathrm{~km} \mathrm{~s}^{-1}$. This result is not compatible with the firm upper limit of $5 \mathrm{~km} \mathrm{~s}^{-1}$ established for $v \sin i$ in the spectroscopic analysis (see Sect. 2.2).

\section{Can ridge $\mathrm{C}$ correspond to $\ell=0$ modes?}

As was said in Sect. 5.1, for the sake of completeness and in the light of previous analyses of p-mode oscillations in solar-like pulsators, we decided to also investigate the alternate identification. In this case, ridges $\mathrm{C}$ and $\mathrm{D}$ correspond to $\ell=0$ and $\ell=2$ modes, respectively. Ridge A could not possibly be an $\ell=3$ ridge, because it has much too strong amplitudes. Ridges A and B would then be the $m= \pm 1$ components of rotationally splitted $\ell=1$ modes.

We fitted the modes exactly the same way it was done before. The results are given in Tables 6 and 7, and an échelle diagram of the fitted modes is shown in Fig. 14. The linewidths, heights and amplitudes are very comparable with those obtained in the first identification. We obtain a splitting of $\nu_{\mathrm{s}}=2.2 \pm 0.1 \mu \mathrm{Hz}$ and an angle of $i=70.6 \pm 5.8^{\circ}$, which is consistent with the idea that ridges $\mathrm{A}$ and $\mathrm{B}$ are indeed identified as $(\ell=1, m= \pm 1)$ modes because they are separated by about twice the fitted splitting. Using Eq. (5) as before, these fitted values of the splitting and angle give an estimate of $v \sin i=17.7 \pm 2.8 \mathrm{~km} \mathrm{~s}^{-1}$, in complete disagreement with the measured $(v \sin i)_{\text {measured }}=2.9_{-1.5}^{+1.0} \mathrm{~km} \mathrm{~s}^{-1}$.

The alternate identifications we considered lead to major disagreements with the measured $v \sin i$ and were therefore rejected. 


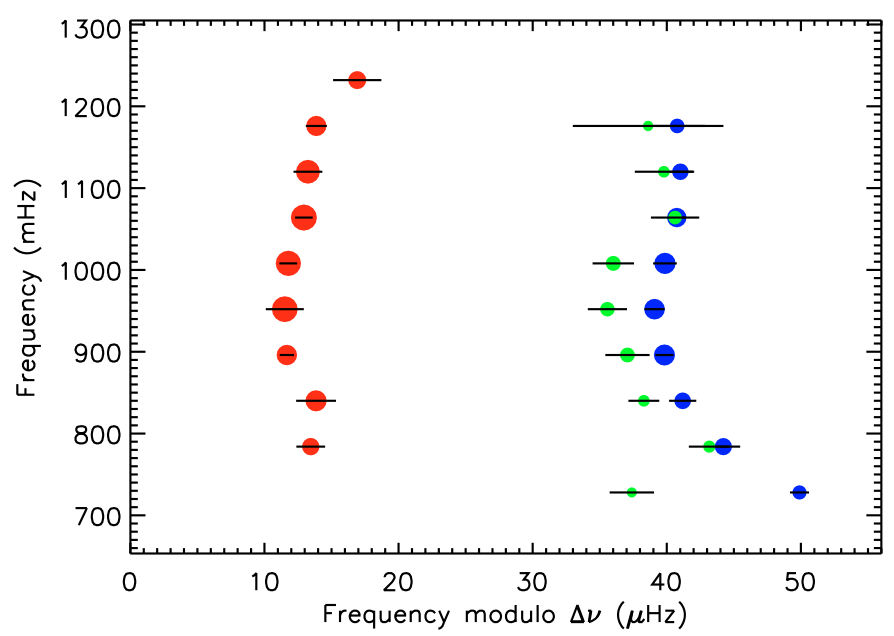

Fig. 14. Échelle diagram for the fitted modes of the alternate identification. Same symbols as in Fig. 10.

Table 7. Reference results for the parameters of the non-radial p modes for the alternate identification (symbols are the same as in Table 3).

\begin{tabular}{cccc}
\hline \hline$\ell$ & $v_{n, \ell}(\mu \mathrm{Hz})$ & agr. $\left(v_{n, \ell}\right)$ & $H_{\ell, n} / B\left(v_{n, \ell}\right)$ \\
\hline 1 & $797.45 \pm 0.36$ & $46.0 \sigma$ & 4.22 \\
1 & $853.85 \pm 0.49$ & $\bullet$ & 4.39 \\
1 & $907.67 \pm 0.18$ & $\bullet$ & 6.56 \\
1 & $963.52 \pm 0.47$ & $\bullet$ & 8.05 \\
1 & $1019.78 \pm 0.22$ & $\bullet$ & 8.36 \\
1 & $1076.94 \pm 0.22$ & $\bullet$ & 10.49 \\
1 & $1133.24 \pm 0.36$ & $\bullet$ & 7.53 \\
1 & $1189.87 \pm 0.26$ & $\bullet$ & 5.15 \\
1 & $1248.92 \pm 0.60$ & $\bullet$ & 2.44 \\
\hline 2 & $765.39 \pm 0.55$ & $39.7 \sigma$ & 1.36 \\
2 & $827.17 \pm 0.51$ & $27.2 \sigma$ & 1.43 \\
2 & $878.29 \pm 0.38$ & $\bullet$ & 2.13 \\
2 & $933.07 \pm 0.55$ & $\bullet$ & 2.62 \\
2 & $987.57 \pm 0.49$ & $\bullet$ & 2.73 \\
2 & $1044.01 \pm 0.52$ & $\bullet$ & 3.43 \\
2 & $1104.62 \pm 0.60$ & $\bullet$ & 2.47 \\
2 & $1159.79 \pm 0.72$ & $\bullet$ & 1.69 \\
2 & $1214.61 \pm 1.87$ & $\bullet$ & 0.80 \\
\hline
\end{tabular}

This confirms the identification we adopted in Sect. 5.2 to perform the global fit of the p-mode parameters.

\section{Conclusion}

The star HD 49385 was characterized from both CoRoT seismic observations and NARVAL spectroscopic data. The atmospheric parameters of the star were derived and the 137-day-long photometric time series was analyzed.

A clear series of peaks associated to $p$ modes was detected in the power spectrum around $1 \mathrm{mHz}$. Up to now, the CoRoT solarlike pulsators presented some ambiguities in the identification of ridges corresponding to different degrees $\ell$. Here three very clear ridges appear in the échelle diagram, which were readily identified as $\ell=0,1$ and 2 modes. The $\ell=2$ ridge appears clearly distinct from the $\ell=0$ ridge in the échelle diagram. Furthermore, two peaks, part of a fainter ridge, were found to be significant and compatible with the characteristics we expect for $\ell=3$ modes in HD 49385. This probably constitutes the first photometric detection of $\ell=3$ modes in a solar-like pulsating star (other than the Sun).

We performed a global fit over nine radial orders with degrees ranging from $\ell=0$ to $\ell=3$ modes (simultaneous fit of 36 individual modes). We obtained precise estimates of the mode frequencies (uncertainty of about $0.2 \mu \mathrm{Hz}$ for radial modes at the maximum of the signal). The obtained value of the maximum amplitude for $\ell=0$ modes $(5.6 \pm 0.8 \mathrm{ppm})$ is consistent with the estimate deduced from Samadi et al. (2007b), unlike the other CoRoT solar-like pulsators (e.g. Appourchaux et al. 2008).

We found no evidence of a rotational splitting of the modes. This can be explained either by a small inclination angle or by a low rotational velocity (inducing a small rotational splitting compared to the linewidth of the modes). In passing we stress that even for very slow rotators (rotational splitting as low as a few $\mu \mathrm{Hz}$ ) the $m \neq 0$ components of non-radial multiplets are not expected to be symmetrical with respect to the $m=0$ component. We proposed a simple way of treating this assymetry in future analyses of solar-like pulsators.

The $\mathrm{p}$ modes of HD 49385 were found to have lifetimes ranging from about one day to two days, i.e. somewhat shorter than the mode lifetimes in the Sun, but significantly larger than those of the previously observed CoRoT solar-like targets. This explains why the spectrum of HD 49385 is much clearer than for previous CoRoT pulsators, for which the large mode linewidths made it harder to separate the $\ell=2$ ridge from the $\ell=0$ ridge. The results obtained for HD 49385 confirm that the linewidths of the modes in G-type pulsators are smaller than those of F-type pulsators, making their analysis easier.

The very high quality of the spectrum also enabled us to detect significant peaks outside the identified ridges. These peaks were found to be compatible with mixed modes, whose presence can be expected in the spectrum of evolved objects such as HD 49385. The existence of mixed modes in avoided crossing can explain some specific behaviors we observe in the lowfrequency eigenmodes. In particular we found that the $\ell=1$ ridge is distorted compared to the $\ell=0$ ridge at low frequency. Deheuvels \& Michel (2009) showed that this type of distortion could be associated with a low-degree mixed mode in avoided crossing. The identification of the mode $\pi_{1}$ as an $\ell=1$ mixed mode would be consistent with the observed pattern. Other features like the $\ell=2$ mode found to overlap the $\ell=0$ mode (around $855 \mu \mathrm{Hz}$ ) might also result from avoided crossing phenomena. This needs to be further investigated in the seismic interpretation of this star.

Acknowledgements. This work was supported by the Centre National d'Etudes Spatiales (CNES). We thank M.-A. Dupret for providing estimates of the mode linewidths for HD 49385, computed with the non-adiabatic pulstion code MAD. I.W. Roxburgh and G. Verner thank the UK Science and Technology Facilities Council for support under grant PP/E001793/1

\section{Appendix A: Estimate of the expected amplitudes for the oscillations in HD 49385}

Scaling laws give an estimate of the amplitude of $\ell=0$ modes at the maximum of the signal. Kjeldsen \& Bedding (1995) 
established the following relation between the luminosity amplitude $A$ of the oscillation and the velocity amplitude $v_{\text {osc }}$ :

$A \equiv \frac{\delta L}{L} \propto \frac{v_{\text {osc }}}{\sqrt{T_{\text {eff }}}}$.

We assume that the frequency at the maximum of the signal $v_{\max }$ scales as the acoustic cutoff frequency $v_{\mathrm{c}}$, i.e.

$\nu_{\max } \propto \frac{g}{\sqrt{T_{\text {eff }}}} \propto \frac{M}{R^{2} \sqrt{T_{\text {eff }}}}$.

Samadi et al. (2007b) obtained

$v_{\mathrm{osc}} \propto\left(\frac{L}{M}\right)^{0.7} \propto\left(\frac{R^{2} T_{\mathrm{eff}}{ }^{4}}{M}\right)^{0.7}$.

Inserting Eqs. (A.2) and (A.3) into Eq. (A.1) we obtain

$A=A_{\odot}\left(\frac{T_{\text {eff }}}{T_{\odot}}\right)^{1.95}\left(\frac{v_{\max }}{v_{\max \odot}}\right)^{-0.7}$.

With the spectroscopic measure of the temperature $T_{\text {eff }}=6095 \pm$ $50 \mathrm{~K}$ and our estimate $v_{\max }=1013 \pm 3 \mu \mathrm{Hz}$ we obtain the expected amplitude for $\ell=0$ modes at the signal maximum $A_{\ell=0}=6.1 \pm 0.5 \mathrm{ppm}$.

\section{Appendix B: Computation of the posterior probability}

By applying the Bayes theorem and assuming an equiprobability of $\mathrm{H}_{0}$ and $\mathrm{H}_{1}$, Appourchaux et al. (2009) obtained the following expression for the posterior probability of the $\mathrm{H}_{0}$ hypothesis given the observed data:

$$
P\left(H_{0} \mid x\right)=\left(1+\frac{P\left(x \mid H_{1}\right)}{P\left(x \mid H_{0}\right)}\right)^{-1} \text {. }
$$

The expression of $P\left(x \mid H_{0}\right)$ for a spectrum binned over $n$ bins is given in Appourchaux (2004)

$P\left(x \mid H_{0}\right)=\frac{x^{n-1} \mathrm{e}^{-x}}{\gamma(n)}$,

where $\gamma(n)$ is the Gamma function. To solve Eq. (B.1), we need to define the alternate hypothesis $\mathrm{H}_{1}$ and to make prior assumptions on the expected signal.

Among the four studied peaks, two are assumed to be $\ell=3$ modes around the maximum of the signal $\left(\pi_{2}\right.$ and $\left.\pi_{3}\right)$. To obtain an expression for $P\left(x \mid H_{1}\right)$, we need an estimate of the expected height and linewidth of $\ell=3$ modes.

An estimate of the expected mode linewidths of HD 49385 was computed with the non-adiabatic pulsation code MAD combined with a $1 \mathrm{D}$ model reproducing the effective temperature, the luminosity and the $\log g$ of the object in the same way as done in Appourchaux et al. (2009). We obtain around the maximum of the signal a linewidth between $1 \mu \mathrm{Hz}$ and $2.5 \mu \mathrm{Hz}$. We adopt a uniform prior for the linewidth, taking into account an uncertainty factor in the theoretical model: we assume that the maximum linewidth is twice as large as that given from the models $(\Gamma \in[0,5] \mu \mathrm{Hz})$.

The height of the $\ell=3$ modes can be deduced from the amplitude of $\ell=0$ modes through the relation

$H_{\ell, m}=r_{\ell, m}(i) H_{\ell=0}=r_{\ell, m}(i) \frac{A_{\ell=0}^{2}}{\pi \Gamma}$ for an angle of inclination $i$. The visibility factors $r_{\ell, m}(i)$ are computed from Gizon \& Solanki (2003). The expected amplitude of the $\ell=0$ modes around the maximum of the signal is computed in Appendix A. We impose a uniform prior for the amplitude of the $\ell=0$ modes $\left(A \in\left[A_{\min }, A_{\max }\right]\right.$, where $A_{\min }$ and $A_{\max }$ are the theoretical values of $A_{\ell=0}$ at $\left.\pm 3 \sigma\right)$ and for the angle of inclination $(i \in[0, \pi / 2])$.

Based on Appourchaux et al. (2009) we obtain the following expression for the probability of the observed data given the signal

$P\left(x \mid H_{1}\right)=\frac{1}{\eta} \int_{A_{\min }}^{A_{\max }} \int_{0}^{\Gamma_{\max }} \int_{0}^{\pi / 2} \frac{\lambda^{v}}{\gamma(v)} x^{\nu-1} \mathrm{e}^{-\lambda x} \mathrm{dA}^{\prime} \mathrm{d} \Gamma^{\prime} \mathrm{di}^{\prime}$,

where $\eta=\Gamma_{\max }\left(A_{\max }-A_{\min }\right) \pi / 2$ is the normalization factor. $\lambda$ and $v$ are given in Appourchaux (2004) and are functions of the mode height $H$ and linewidth $\Gamma$.

Inserting Eqs. (B.2) and (B.4) into Eq. (B.1), we obtain for $\pi_{2}$ and $\pi_{3}$ a posterior probability of $1.4 \%$ and $1.1 \%$, respectively.

Two peaks are assumed to be the signature of mixed modes ( $\pi_{1}$ and $\pi_{4}$ ). For this type of modes, a part of the energy is located in the g-mode cavity and the inertia is larger. We therefore expect a smaller linewidth for these modes. But we can have no clue of their degree $\ell$. Because both their amplitudes and their linewidths greatly depend on $\ell$, we use very conservative priors: uniform priors on the amplitude of the mode $\left(A \in\left[0, A_{\max }\right] \mathrm{ppm}\right)$ and on the linewidth $(\Gamma \in[0,5] \mu \mathrm{Hz})$.

We obtain posterior probabilities of $0.001 \%$ and $8.7 \%$ for $\pi_{1}$ and $\pi_{4}$, respectively.

\section{References}

Anderson, E. R., Duvall, Jr., T. L., \& Jefferies, S. M. 1990, ApJ, 364, 699 Appourchaux, T. 2004, A\&A, 428, 1039

Appourchaux, T., Gizon, L., \& Rabello-Soares, M.-C. 1998, A\&AS, 132, 107 Appourchaux, T., Michel, E., Auvergne, M., et al. 2008, A\&A, 488, 705 Appourchaux, T., Samadi, R., \& Dupret, M. A. 2009, A\&A, 506, 1 Asplund, M. 2005, ARA\&A, 43, 481

Auvergne, M., Bodin, P., Boisnard, L., et al. 2009, A\&A, 506, 411

Baglin, A., Auvergne, M., Barge, P., et al. 2006, in ESA SP 1306, ed. M. Fridlund, A. Baglin, J. Lochard, \& L. Conroy, 33

Ballot, J., García, R. A., \& Lambert, P. 2006, MNRAS, 369, 1281

Barban, C., Deheuvels, S., Baudin, F., et al. 2009, A\&A, 506, 51

Basu, S., Christensen-Dalsgaard, J., Howe, R. et al. 2003, ApJ, 591, 432

Bedding, T. R., \& Kjeldsen, H. 2010, Commun. Asteroseismol., 161, 3

Bessell, M. S., Castelli, F., \& Plez, B. 1998, A\&A, 333, 231

Boesgaard, A. M., Stephens, A., \& Deliyannis, C. P. 2005, ApJ, 633, 398

Bruntt, H. 2009, A\&A, 506, 235

Bruntt, H., De Cat, P., \& Aerts, C. 2008, A\&A, 478, 487

Christensen-Dalsgaard, J. 2003, Lect. Not. Stellar Oscillat.

Christensen-Dalsgaard, J., Gough, D. O., \& Thompson, M. J. 1991, ApJ, 378, 413

Deheuvels, S., \& Michel, E. 2009, Ap\&SS, 241

Duvall, Jr., T. L., \& Harvey, J. W. 1986, in NATO ASIC Proc. 169: Seismology of the Sun and the Distant Stars, ed. D. O. Gough, 105

Dziembowski, W. A., \& Pamyatnykh, A. A. 1991, A\&A, 248, L11

García, R. A., Régulo, C., Samadi, R., et al. 2009, A\&A, 506, 41

Ghezzi, L., Cunha, K., Smith, V. V., et al. 2009, ApJ, 698, 451

Gizon, L., \& Solanki, S. K. 2003, ApJ, 589, 1009

Harvey, J. 1985, in Future Missions in Solar, Heliospheric \& Space Plasma Physics, ed. E. Rolfe \& B. Battrick, ESA SP, 235, 199

Hauck, B., \& Mermilliod, M. 1998, A\&AS, 129, 431

Kjeldsen, H., \& Bedding, T. R. 1995, A\&A, 293, 87

Kovtyukh, V. V., Soubiran, C., \& Belik, S. I. 2004, A\&A, 427, 933

Kupka, F., \& Bruntt, H. 2001, Using TEMPLOGG for determining stellar parameters of MONS targets, ed. C. Sterken (C), 39

Kurucz, R. L., Furenlid, I., Brault, J., \& Testerman, L. 1984, Solar flux atlas from 296 to 1300 nm, ed. R. L. Kurucz, I. Furenlid, J. Brault, \& L. Testerman 
S. Deheuvels et al.: Seismic and spectroscopic analysis of solar-like pulsating CoRoT target HD 49385

Mathur, S., Eff-Darwich, A., García, R. A., \& Turck-Chièze, S. 2008, A\&A, 484, 517

Mathur, S., García, R. A., Régulo, C., et al. 2010, A\&A, 511, A46

Michel, E., Baglin, A., Auvergne, M., et al. 2008, Science, 322, 558

Michel, E., Samadi, R., Baudin, F., et al. 2009, A\&A, 495, 979

Morel, P. 1997, A\&AS, 124, 597

Mosser, B., \& Appourchaux, T. 2009, A\&A, 508, 877

Mosser, B., Baudin, F., Lanza, A. F., et al. 2009a, A\&A, 506, 245

Mosser, B., Michel, E., Appourchaux, T., et al. 2009b, A\&A, 506, 33

Ramírez, I., \& Meléndez, J. 2005, ApJ, 626, 465

Rogers, N. Y. 1995, Commun. Asteroseismol., 78, 1

Samadi, R., Fialho, F., Costa, J. E. S., et al. 2007a, [astro-ph/0703354]
Samadi, R., Georgobiani, D., Trampedach, R., et al. 2007b, A\&A, 463, 297 Scuflaire, R. 1974, A\&A, 36, 107

Suárez, J. C., \& Goupil, M. J. 2008, Ap\&SS, 316, 155

Thompson, M. J., Christensen-Dalsgaard, J., Miesch, M. S., \& Toomre, J. 2003, ARA\&A, 41, 599

Torrence, C., \& Compo, G. P. 1998, Bull. Am. Meteorol. Soc., 79, 61

Toutain, T., \& Froehlich, C. 1992, A\&A, 257, 287

Turck-Chièze, S., Couvidat, S., Kosovichev, A. G., et al. 2001, ApJ, 555, L69

Unno, W., Osaki, Y., Ando, H., Saio, H., \& Shibahashi, H. 1989, Nonradial oscillations of stars (University of Tokyo Press), 2nd ed.

Valenti, J. A., \& Piskunov, N. 1996, A\&AS, 118, 595

van Leeuwen, F. 2007, A\&A, 474, 653 